\title{
Technologies for Optical Processing
}

\author{
Stubkjær, Kristian
}

Published in:

Conference on Optical Fiber communication/National Fiber Optic Engineers Conference, 2008. OFC/NFOEC 2008.

Link to article, DOI:

10.1109/OFC.2008.4528416

Publication date:

2008

Document Version

Publisher's PDF, also known as Version of record

Link back to DTU Orbit

Citation (APA):

Stubkjær, K. (2008). Technologies for Optical Processing. In Conference on Optical Fiber communication/National Fiber Optic Engineers Conference, 2008. OFC/NFOEC 2008. IEEE. https://doi.org/10.1109/OFC.2008.4528416

\section{General rights}

Copyright and moral rights for the publications made accessible in the public portal are retained by the authors and/or other copyright owners and it is a condition of accessing publications that users recognise and abide by the legal requirements associated with these rights.

- Users may download and print one copy of any publication from the public portal for the purpose of private study or research.

- You may not further distribute the material or use it for any profit-making activity or commercial gain

- You may freely distribute the URL identifying the publication in the public portal

If you believe that this document breaches copyright please contact us providing details, and we will remove access to the work immediately and investigate your claim 
Tutorial OFC 2008

Technologies for Optical Processing

Kristian E. Stubkjaer

Technical University of Denmark DK-2800 Lyngby stubkjaer@adm.dtu.dk

\section{Acknowledgements}

Many have helped with material for the presentation including:

- L. Oxenloewe, J. Moerk and A. Bjarklev, F. Öhman, J. Buron, A. Boltasseva, COM

- A. Soerensen, Niels Bohr. Inst., Copenhagen Univ.

- M.L. Nielsen, NEC

- M.K. Smit, E. Tangdiongga and H.J.S. Dorren, TU/e

- D. Blumenthal, UCSB 


\section{Dream of the optical transistor}

Why?

- Keeping signals in the optical domain

- Small losses in transmission

- Long term: excessive processing capacity

Important factors

- Size/compactness

- Switching energy

- Switching time

- Wavelength

- Cost

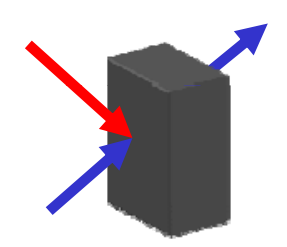

\section{Contents}

- Application areas

- Types of nonlinear gates

- Semiconductor optical amplifier (SOA)

- Interferometers and cavities

- Fibers and waveguides

- All-optical logic and flip-flops

- Towards ultra compact and single photon transistors 
WDM cross connects

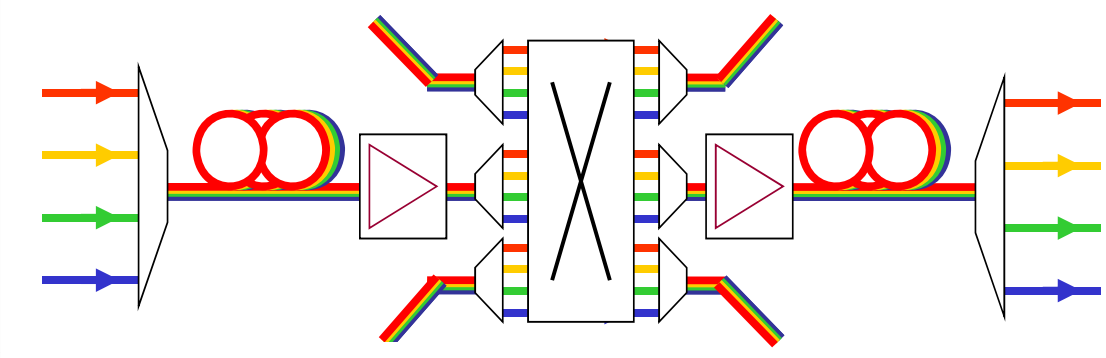

Key components:

- Switches

- Demuxes

-Wavelength converters

\section{Optical Time Division Multiplex Systems}

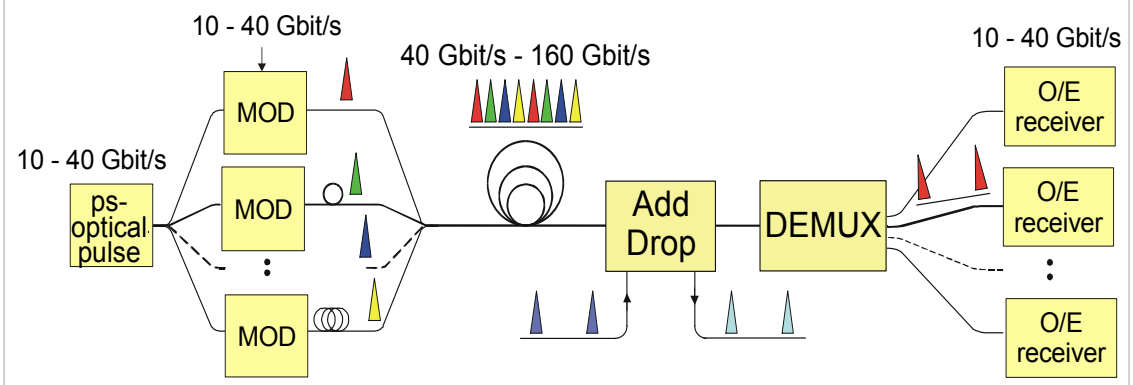

Key components:

- Pulse sources

- Demuxes

- Add/drops 


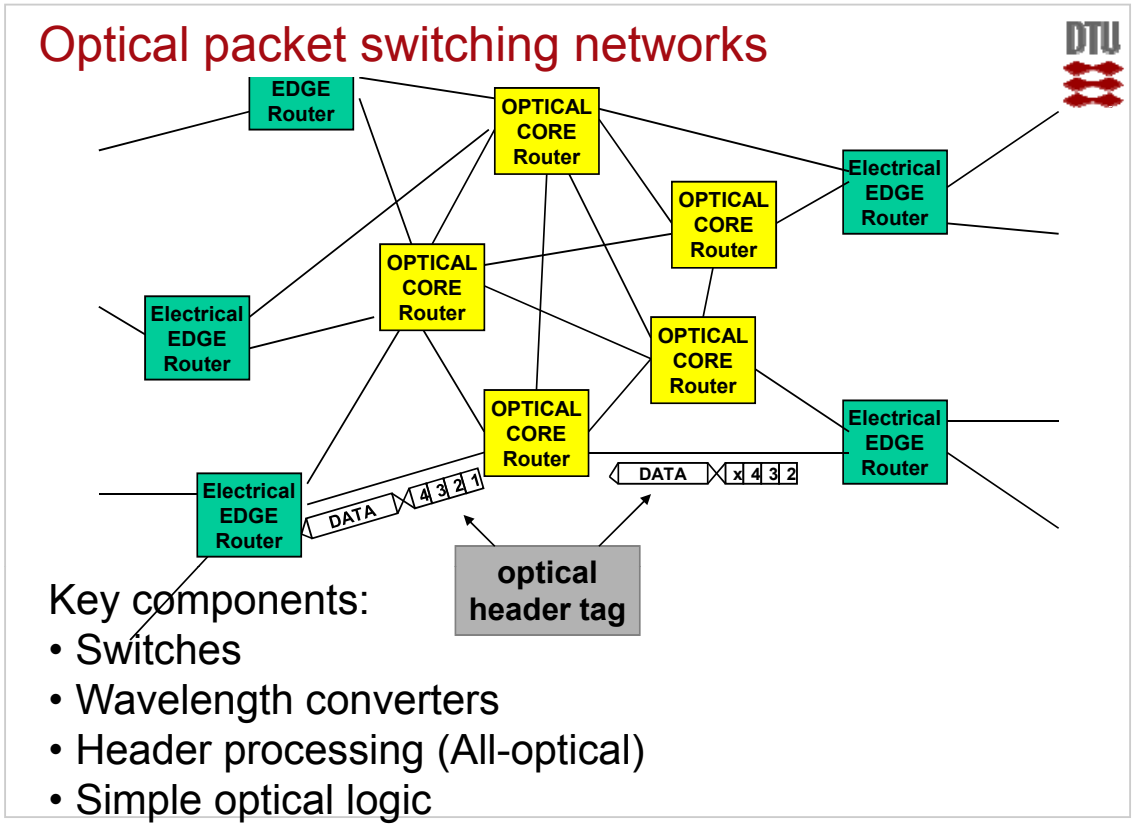

\section{Optical computer (into the future?)}

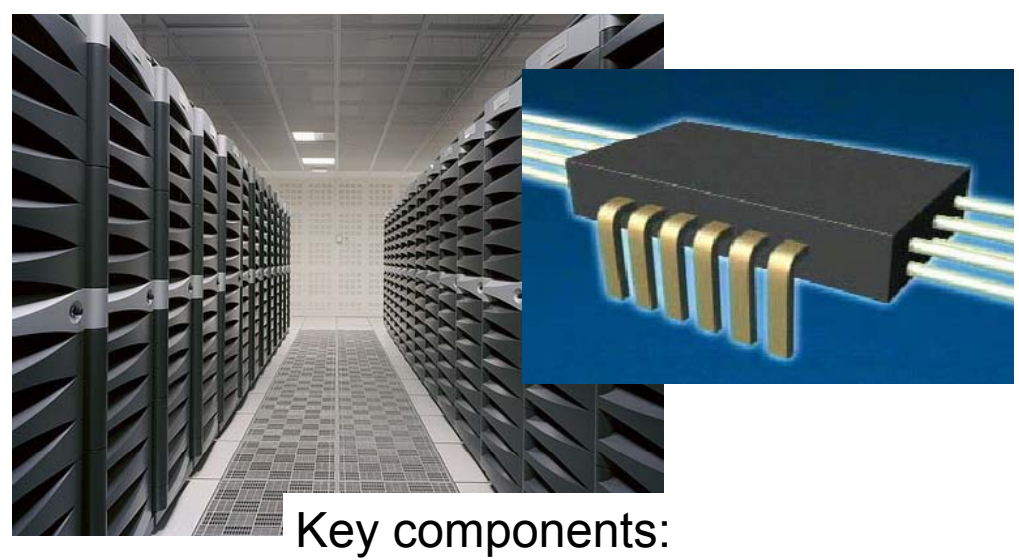

- Optical interconnects

- Massive all-optical logic 


\section{OMV1.pdf}

\begin{tabular}{|c|c|c|c|c|c|}
\hline $\begin{array}{l}\text { State of the art } \\
\text { Commercial }\end{array}$ & 1995 & & 2000 & 2005 & 2010 \\
\hline $\begin{array}{l}\text { Line Bit Rate } \\
\text { Total Fibre Capaci }\end{array}$ & $\operatorname{ci} 20 \begin{array}{r}2.5 \mathrm{~Gb} \\
20-40 \mathrm{Gk}\end{array}$ & $100 \mathrm{~Gb}$ & $\begin{array}{l}2.5 \mathrm{~Gb} \\
10 \mathrm{~Gb} \\
800 \mathrm{~Gb}\end{array}$ & $\begin{array}{l}2.5 \mathrm{~Gb} \\
-10 \mathrm{~Gb} \\
>1 \mathrm{~Tb}\end{array}$ & $\begin{array}{l}2.5 \mathrm{~Gb} \\
-40 \mathrm{~Gb}\end{array}$ \\
\hline \# WDM Channels & 8 & $32-64$ & 128 & 256 & \\
\hline $\begin{array}{l}\text { Channel Spacing } \\
\text { Opt. Transparent }\end{array}$ & $\begin{array}{l}200 \mathrm{GHz} \\
120 \mathrm{~km}\end{array}$ & $\begin{array}{l}\mathrm{z} \quad 100 \mathrm{GH}_{\mathrm{i}} 50 \\
600 \mathrm{~km}\end{array}$ & $\begin{array}{l}0 \mathrm{GHz} \\
\quad 3-5000 \mathrm{~km}\end{array}$ & $5000+\mathrm{km}$ & $10000+\mathrm{km}$ \\
\hline $\begin{array}{l}\text { Network } \\
\text { Architecture }\end{array}$ & $\begin{array}{l}\text { pt-pt } \\
\text { WDM }\end{array}$ & $\left\langle\begin{array}{c}\text { fixed } \\
\text { QADM/ring }\end{array}\right.$ & $\left\langle\begin{array}{c}\text { flexible OADM/ } \\
\text { interconnected) rings }\end{array}\right.$ & $\begin{array}{l}\text { small meshed, flexibjle } \\
\text { WDM networks }\end{array}$ & $\begin{array}{l}\text { meshed, flexible } \\
\text { WDM networks }\end{array}$ \\
\hline \multirow[t]{3}{*}{ Subsystems } & $\mathrm{OA}$ & $\begin{array}{l}\text { fixed } \\
\text { OADM }\end{array}$ & $\begin{array}{l}\text { flexible OADM } \\
\text { small OXC }\end{array}$ & \multicolumn{2}{|c|}{$\begin{array}{l}\text { large, full flexible } \\
\text { (integrated) } \mathrm{nv}=\end{array}$} \\
\hline & & \multirow{2}{*}{\begin{tabular}{|c|}
$3 R$ O/E/O \\
Transponders \\
\end{tabular}} & AOWC & \multirow{2}{*}{\begin{tabular}{|c|} 
integrated \\
optical switches \\
\end{tabular}} & Optical signal \\
\hline & & & $40 \mathrm{Gbit} / \mathrm{s} \mathrm{ICs}$ & & $\begin{array}{l}\text { processing } \\
\text { - OTDM }\end{array}$ \\
\hline \multirow[t]{2}{*}{ Components } & DFB laser & \multicolumn{2}{|c|}{ selectable/ tuneable lasers } & Opt. 3R & - clock \\
\hline & & \multicolumn{2}{|c|}{ low cost optical transceivers } & lower cost opt transc. & \begin{tabular}{|l} 
extraction \\
- short pulse
\end{tabular} \\
\hline $\begin{array}{l}\text { User Access } \\
\text { Bit Rate }\end{array}$ & $\begin{array}{l}\text { POTS } \\
64 \mathrm{~Kb}\end{array}$ & $\begin{array}{c}\text { ISDN } \\
128 \mathrm{~Kb}, 2 \mathrm{Mb}\end{array}$ & $\begin{array}{l}\text { cable modem } \\
\text { ADSL } \\
2-8 \mathrm{Mb}\end{array}$ & $\begin{array}{l}\text { Optical : } 155 \mathrm{Mb} \\
\text { (A|V)DSL at } \\
2,10,50 \mathrm{Mb} \\
\end{array}$ & $\begin{array}{l}\text { Opticair } \\
\text { Mb Electrical: } \\
\text { up to } 100 \mathrm{Mb}\end{array}$ \\
\hline Services & POTS & $\begin{array}{l}\text { Internet, } \\
\text { Videophony, } \\
\ldots\end{array}$ & $\begin{array}{l}\text { Teleworking, Lan-Lan, } \\
\text { fast Internet, video, } \\
\text { games,telelearning... }\end{array}$ & $\begin{array}{l}\text { Very fast Internet, } \\
\text { Interactive } \\
\text { entertainment }\end{array}$ & $\begin{array}{l}\text { Virtual presence for } \\
\text { working, learning and } \\
\text { entertainment }\end{array}$ \\
\hline \multicolumn{4}{|c|}{ HORIZON Project - ACTS } & & Nov 1999 \\
\hline
\end{tabular}

\section{Electronic switching}
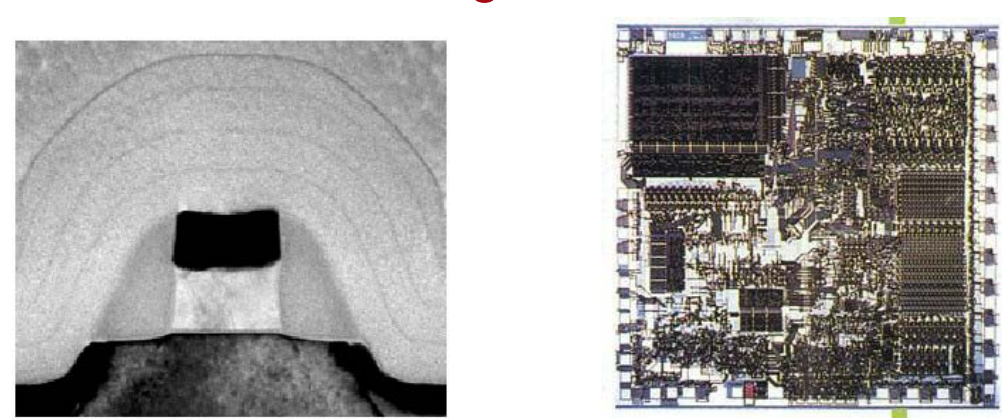

$$
U=\frac{1}{2} C V^{2}
$$

Number is much larger on chip because every wire is also a capacitor;

The energy for an "average"

\section{$1 \mathrm{fJ} /$ switching event} switching event is likely to be 10-100 fJ.

After TF Krauss, http://www.cfn.uni-karlsruhe.de/web/ 
Simple functions with all-optical gates

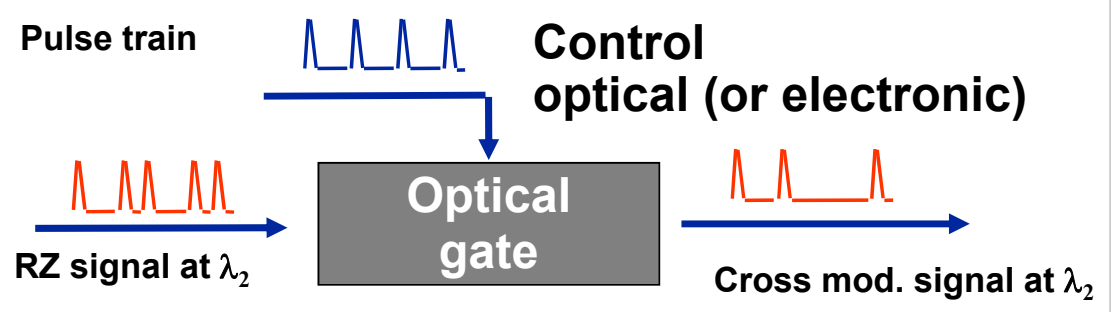

Cross gain/absorption

Cross Phase

Four wave mixing

Difference frequency

generation

Interaction between optical nearfield and electronic states of material

Cross Gain/(Absorption) Modulation (XGM)
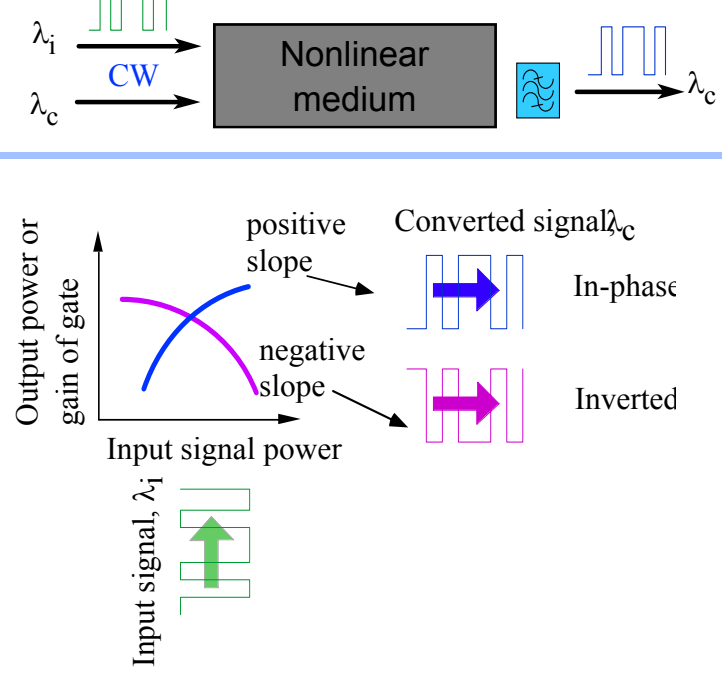


\section{Cross Phase Modulation (XPM)}

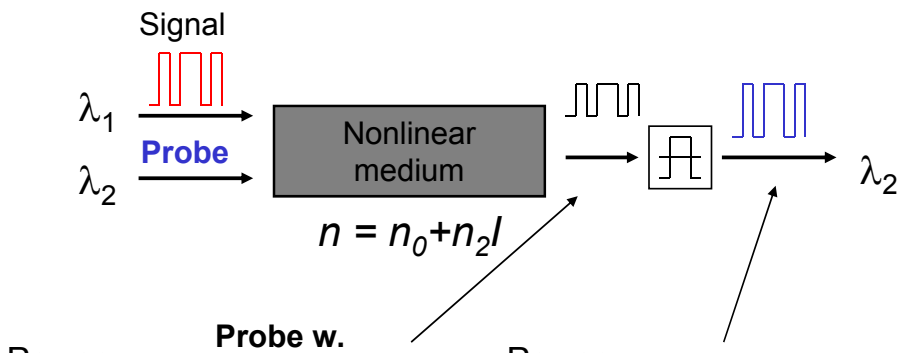

Power XPM sidebands

Power
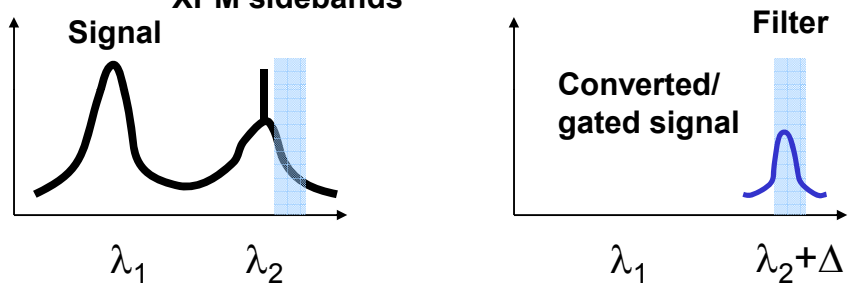
2 


\section{Semiconductor optical amplifier} (SOA) as nonlinear gate

Tested technology

Large band-filling nonlinearity $\left(\mathrm{n}_{2} \sim 10^{6} \mathrm{x}\right.$ silica fiber $)$

Switch powers $<100 f J$

Switching time is an issue

Requires bias of 10-100 mA

Compact (mm size) and compatible with photonic integration

Numerous experimental results

\section{Amplification and saturation in an SOA}
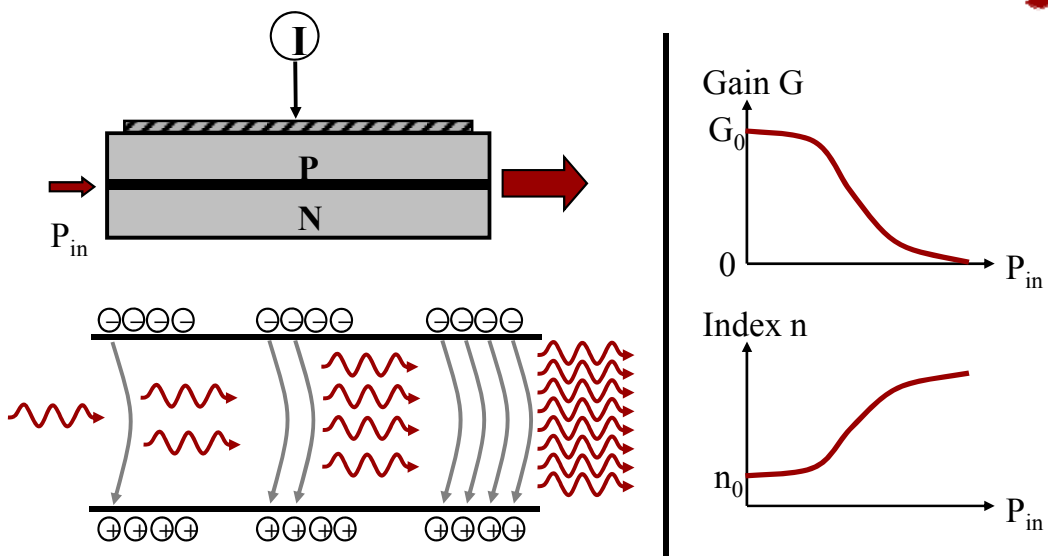

Index $n$

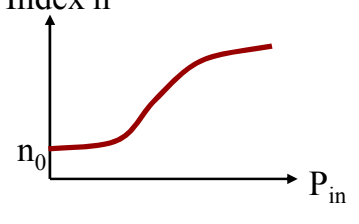

\section{Saturation allows control of light with light -}

but at which speed? 
SOA dynamics - patterning effects

Cross-gain modulation for wavelength conversion: control signal @ $\lambda_{1}$

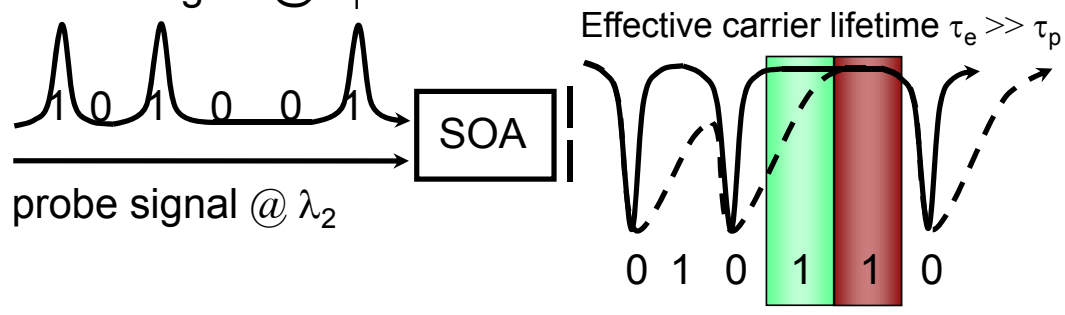

Patterning effect: energy in time slot depends on previous bits

Notice: The phase is modulated analogously through XPM

J. Moerk and M.L. Nielsen, COM

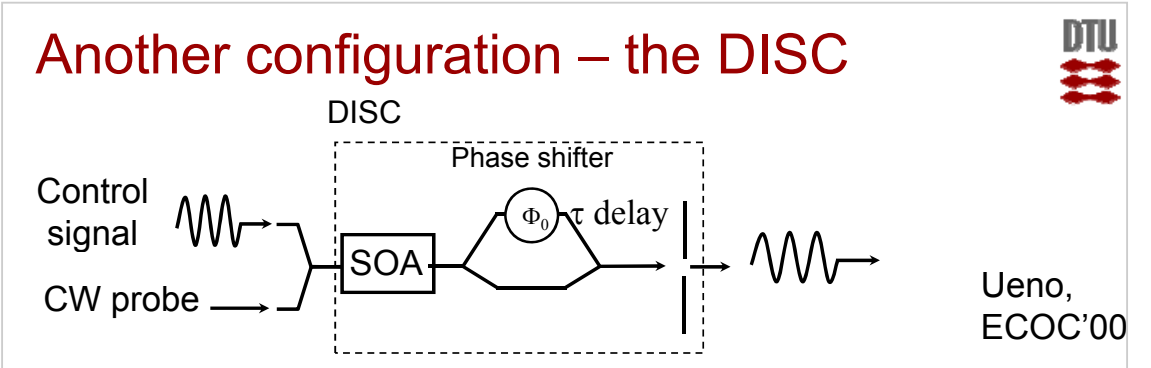

This configuration may be viewed in general as an SOA followed by an external filter:

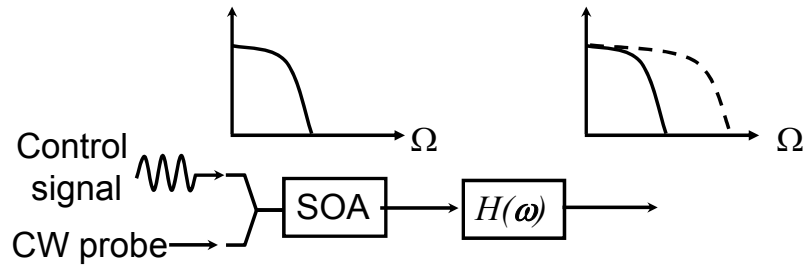

\section{Bandwidth enhancement using optical filtering}

J. Moerk and M.L. Nielsen, COM 


\section{Effect of filtering}

Normalized response at input:

Normalized response at output:

$T_{C D P}(\Omega)=\frac{p_{p}(\Omega)}{p_{p}(0)}=\frac{1}{1-j \Omega \tau_{s}} \rightarrow H(\omega) \rightarrow T_{\text {tot }}(\Omega)=\frac{p_{\text {out }}(\Omega)}{p_{\text {out }}(0)}=T_{H}(\Omega) T_{C D P}(\Omega)$

Transfer function for AMZI:
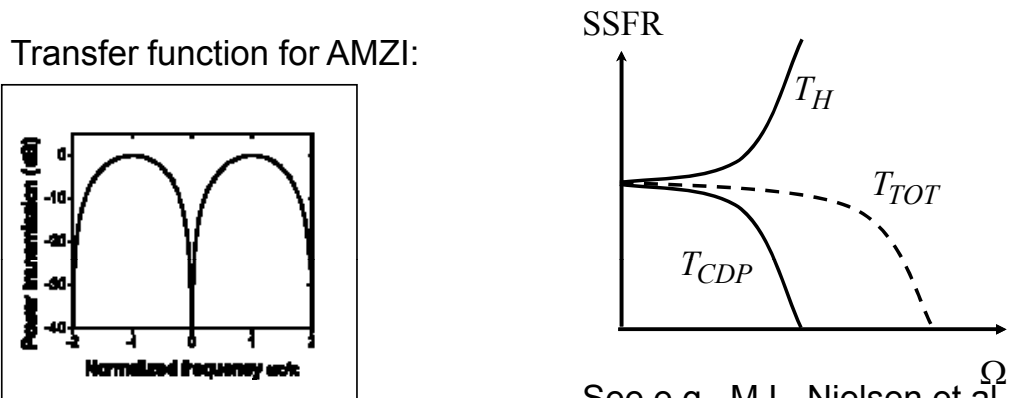

See e.g., M.L. Nielsen et al.,

$\tau$ : Period of transfer function Opt. Exp., Vol. 14, No. 3,

$\Phi_{0}$ : Translates transfer function February 2006.

\section{$160 \mathrm{~Gb} / \mathrm{s} \mathrm{AlJ-Optical} \mathrm{Operatjon}$}

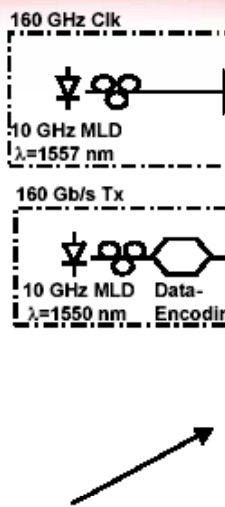

A $160 \mathrm{~Gb} / \mathrm{s} 3 \mathrm{R}$ all-optical wavelength conversion.

Required input power lower than $\mathbf{- 2} \mathrm{dBm}$.

Regeneration for degraded input signals.

Ref.: J. Leuthold et al., to be published in El. Lett, 2004 Courtesy of M. Renaud 

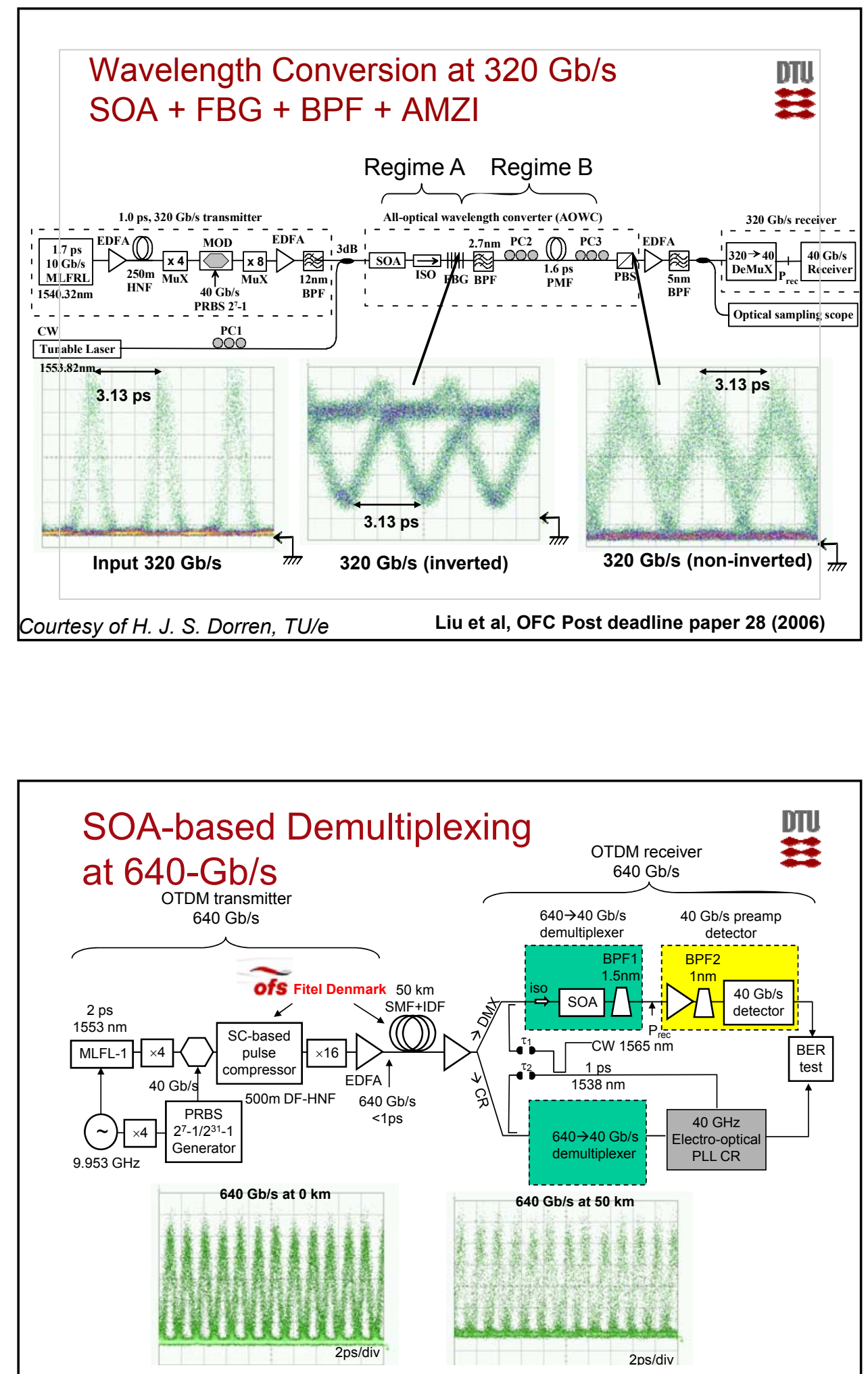

E. Tangdiongga et al., TU/e, Presented at ECOC 2007, Berlin, Sept. 2007 


\section{Differential control scheme}

(S. Nakamura, 1994)

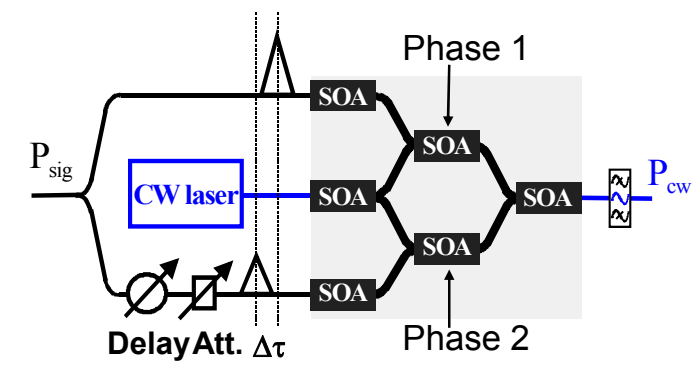

Slow carrier recovery effects removed
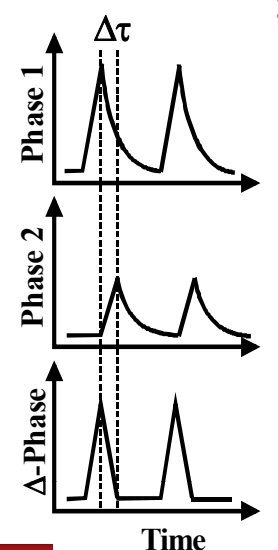

short switching window

\section{Mach-Zehnder Interferometer}

Flip-chip mounted MZI-SOA
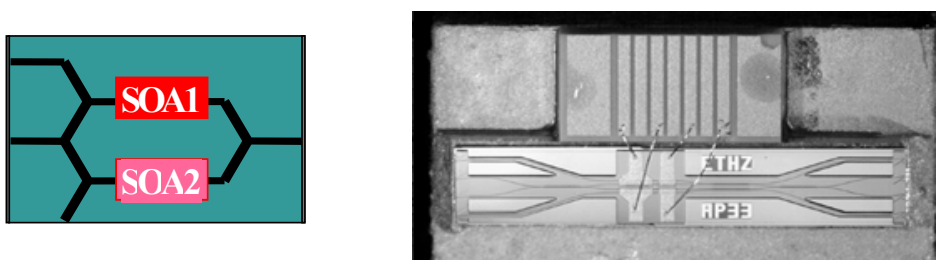

H. Melchior et al., ETH-Zürich Mid-90's 
Ultra high-speed optical signal proc.

w. MZI type switch

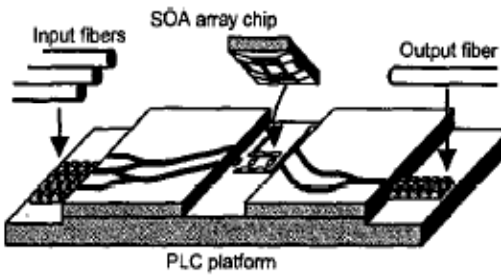

Fig. 1. Hybrid-integrated Symmetric Mach-Zehnder all-optical switch

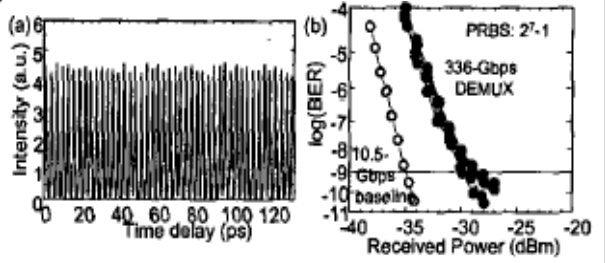

Fig. 2. 336-Gbps demultiplexing with HI-SMZ swilch (a) Cross-correlation trace of input signal pulses. (b) Results of BER measurement.

Nakamura, Ueno and Tajima, NEC IEEE LEOS Summer Topical meeting, 2004
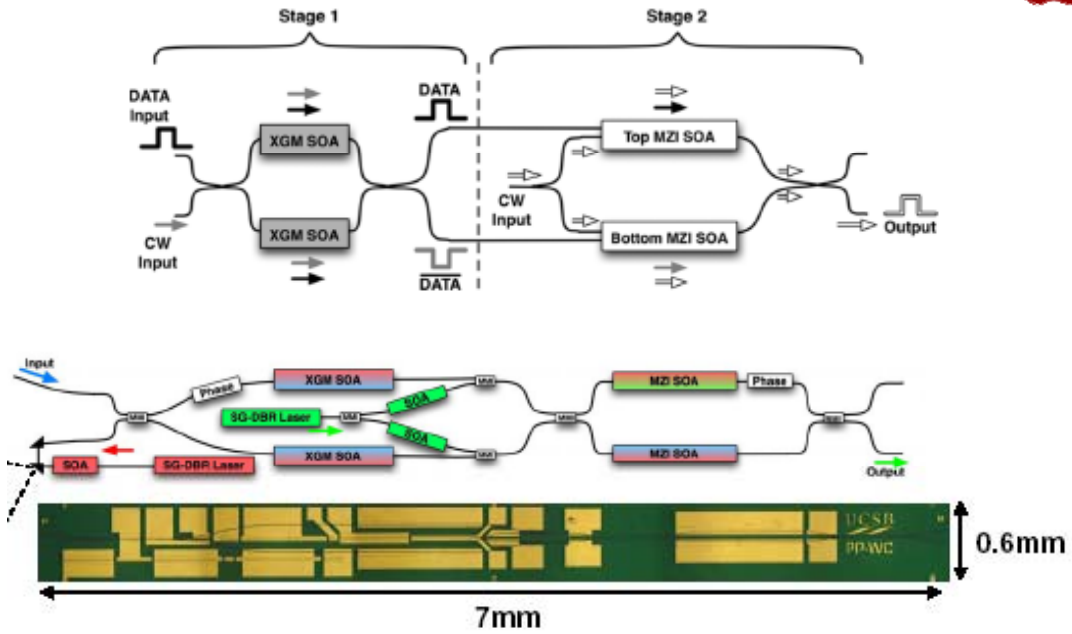

J.A. Summers et al., Photon. Techn. Lett., Vol. 19, No. 21, p. 1768, Nov., 2007 


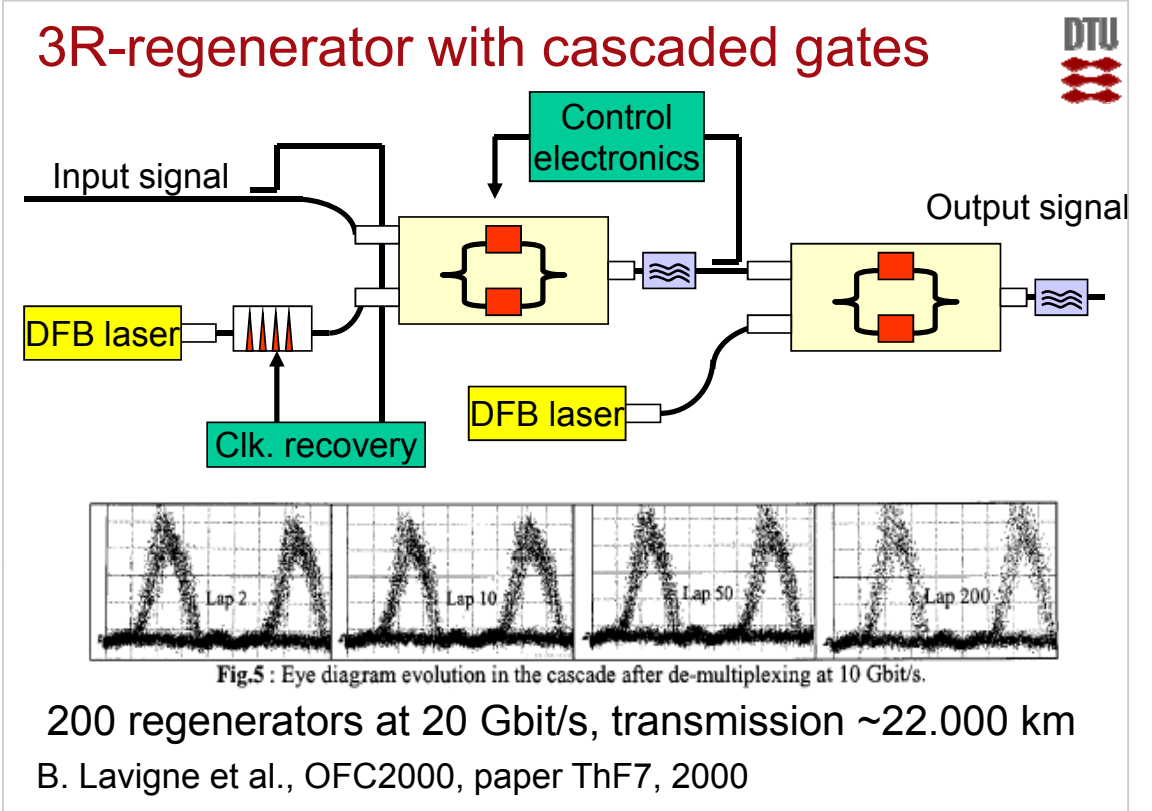

Other interferometric gates UNI

ultrafast nonlinear interferometer

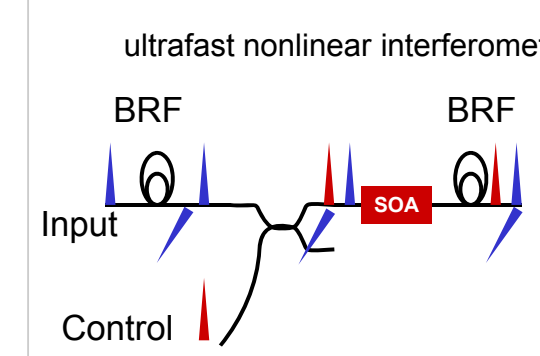

TOAD/SLALOM

et al. (MIT), Photon. Techn. Lett., vol. 8, pp. 1695-1697, 96

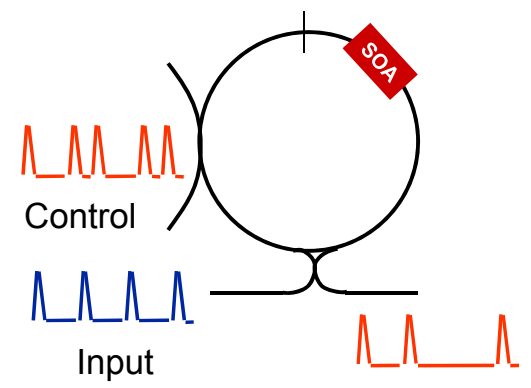

Sokoloff et al. (Princeton), Photon. Techn. Lett., vol. 5, pp.787-790, 93. 


\section{Quantum confinement}

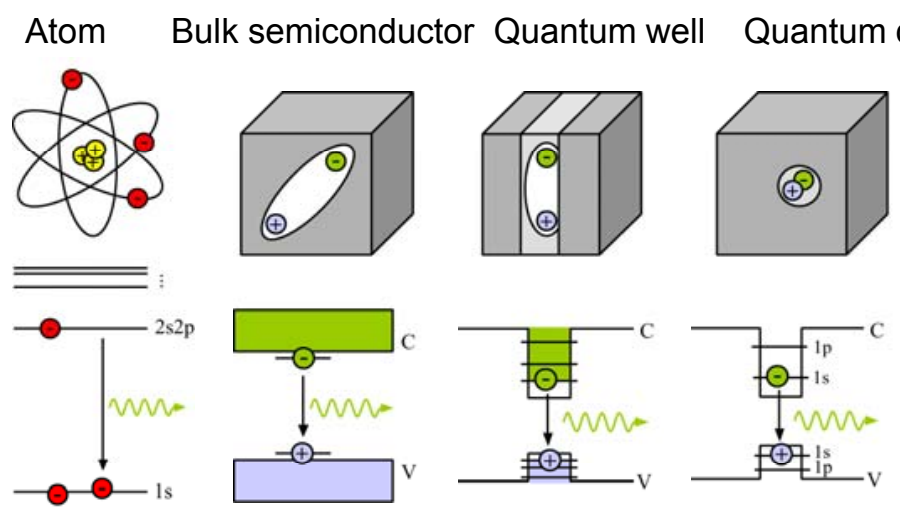

Engineering materials with unique properties:

1. Enhanced interaction with light for more efficient device operation.

2. Reduced scattering for faster dynamics $(\mathrm{GHz} \rightarrow \mathrm{THz})$.

3. Better wavelength control and short pulse propagation (chirp effects).

\section{Signal processing in QD SOAs}

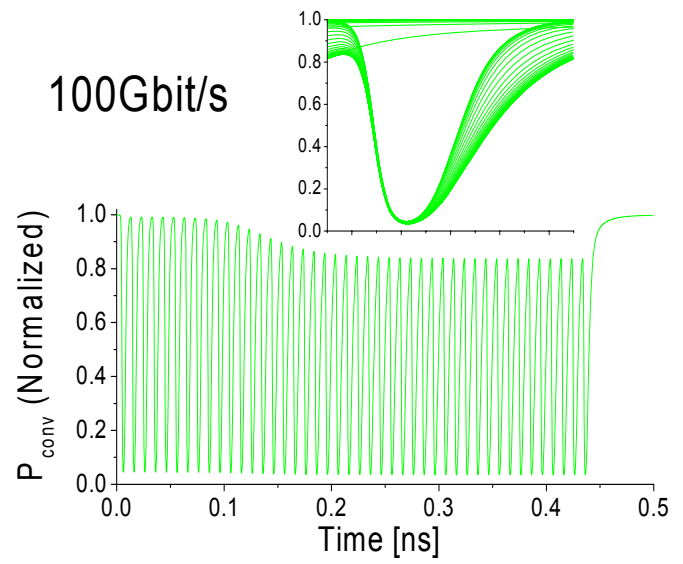

- Long device (4 mm)

- 20dB gain

- 1 pJ input pulses

- 1 ps pulses

- 1 ps capture

- Worst case signal

Berg et al., CLEO'02

Sugawara et al. JJAP, '01

Exp. Demo@ 40 Gb/s:

Akiyama et al., ECOC'02

SHB - fast recovery (capture time)

Fully inverted QDs and large reservoir - small patterning

J. Moerk and M.L. Nielsen, COM 


\section{Photonic crystals and quantum dots}
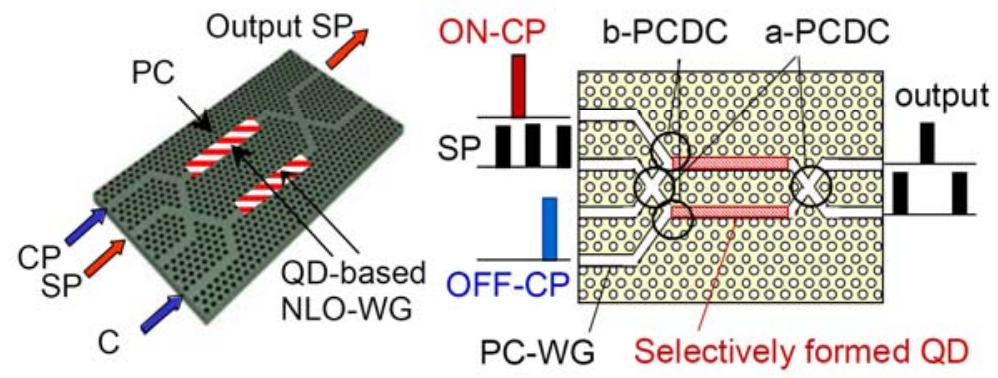

Rise and fall times $\sim 2 \mathrm{ps,}$ switching energies of control pulse as low as $100 \mathrm{fJ}$.

Asakawa et al., New J. Physics (2006)

\section{All-optical intersubband switching}

Optical transitions in QW

C. B.

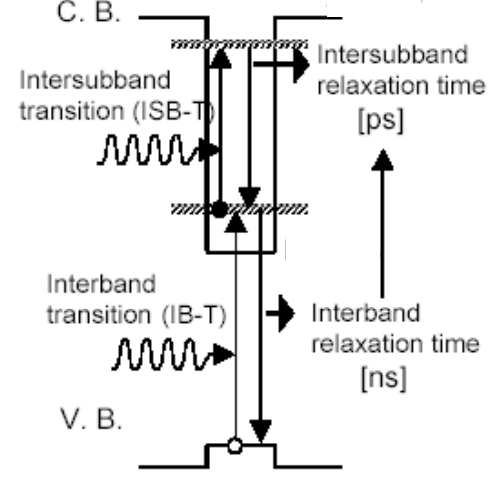

Recently: CdS/ZnSe)/BeTe achieved $<7 \mathrm{pJ}$ switching energy at $1520 \mathrm{~nm}$

G.W. Cong et al., Opt. Exp., 10 Sept. 2007, Vol. 15, No. 19 


\section{DBR-laser converters}

\section{Stimulated recombinations caused by the input signal}

Cross-gain modulation

Decreasing output power

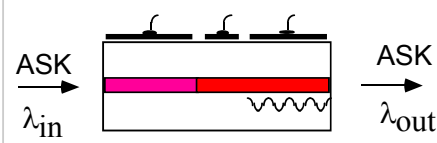

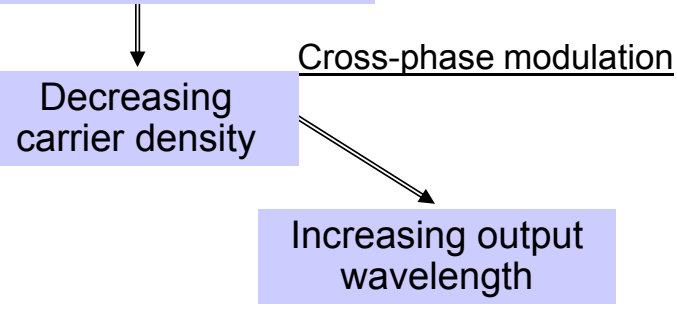

Cross-phase modulation

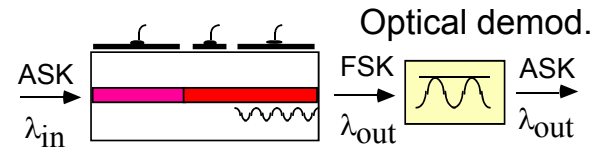

\section{Nano/micro cavity lasers}

(a) 4 pulsed LD

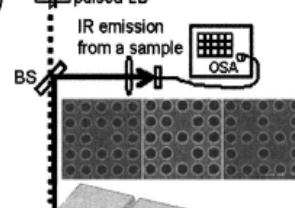

$130 \mathrm{GHz}$ resonances!

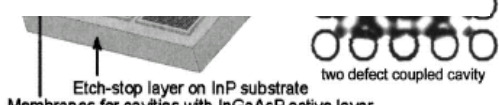

T. Yoshie et al.,

Appl. Phys. Lett.,

Vol. 84 , No. 18 ,

May 2004

(b)

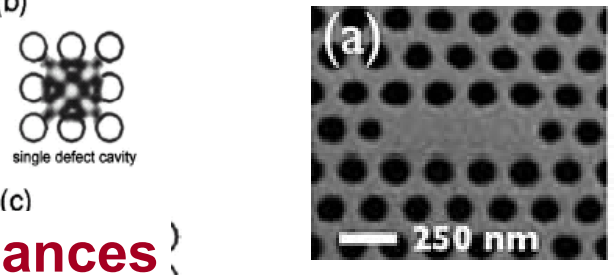

$60 \mathrm{fJ}, 20 \mathrm{GHz}$ tuning by free carrier effect

I. Fushman et al,, Appl. Phys. Lett., Vol. 90, March 2007 


\section{Fibers and waveguides as} nonlinear elements

Fibre switches:

Kerr effect $\chi^{(3)}$ non-linear phase shift: $\phi \sim \gamma \mathrm{PL}$, caused by molecular vibrations $\sim 5 \mathrm{fs}$ response time

Typical control pulse (5ps FWHM at $10 \mathrm{GHz}$ ): $50 \mathrm{~mW}$ average switcing power $\sim 1 \mathrm{~W}$ peak power for commercially available HNLF $(\gamma \sim 10$, $\mathrm{L} \sim 500 \mathrm{~m}$ )

\section{Wavelength conversion in Highly Nonlinear} Fibers by Raman-assisted XPM

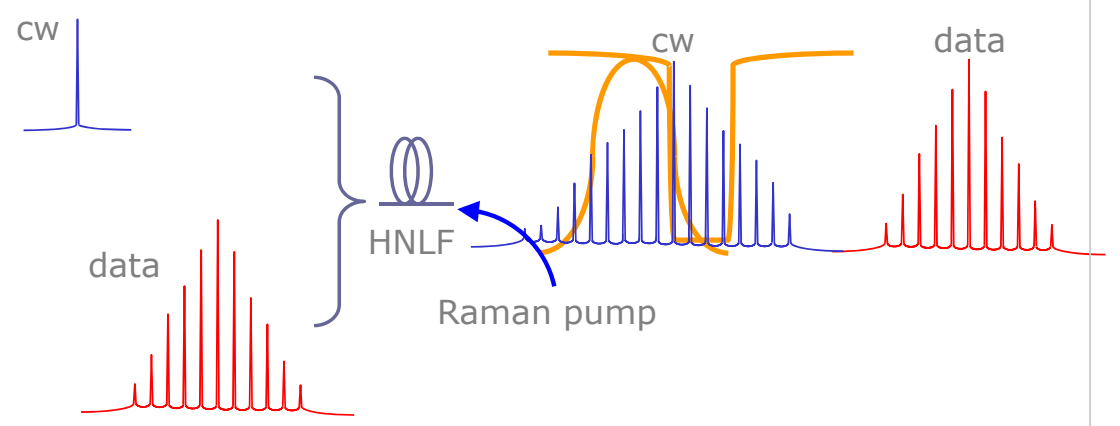

Raman-assisted XPM-based $\lambda$-conversion at $160 \mathrm{~Gb} / \mathrm{s}$ :

- XPM creates sidebands on cw

- Notch-filter suppresses cw and one sideband,

- Bandpass filter removes data

- Raman-effect increases OSNR Michael Galili et al, OFC 2006

L. Oxenloewe 


\section{Photonic crystal fibre demultiplexer}

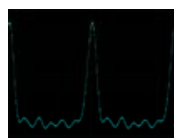

NOLM
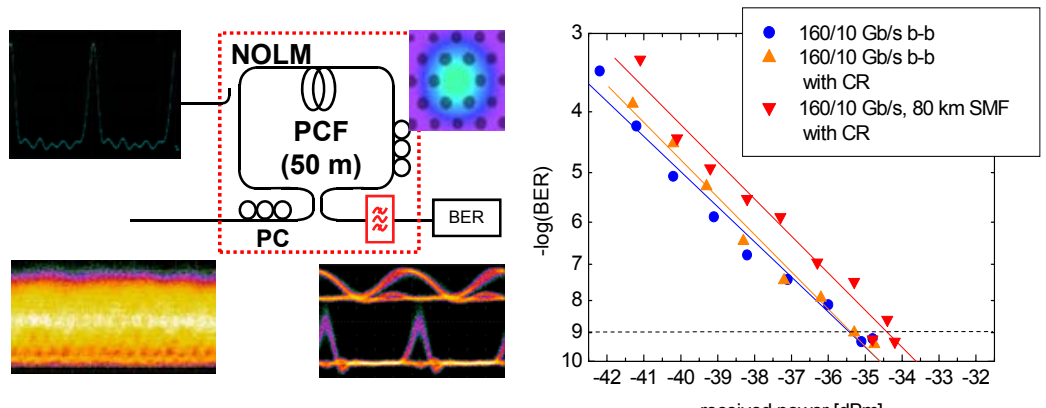

PC

(50 m) 8

PC

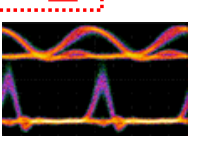

received power $[\mathrm{dBm}]$

First 160 to 10 Gbit/s error free demultiplexing with PCF 1,2

Strong confinement $\rightarrow$ high- $\gamma\left(18 \mathrm{~W}^{-1} \mathrm{~km}^{-1}\right) \rightarrow$ short fibre: $50 \mathrm{~m}$

1: L.K. Oxenløwe et al, OECC 2002, paper PD-1-4,

2: L.K. Oxenløwe et al, ECOC 2003, paper Th2.5.3 16E3-5

L.K. Oxenloewe

\section{$640 \mathrm{Gbit} / \mathrm{s}$ demultiplexing}

\section{Separate low jitter pulse sources}
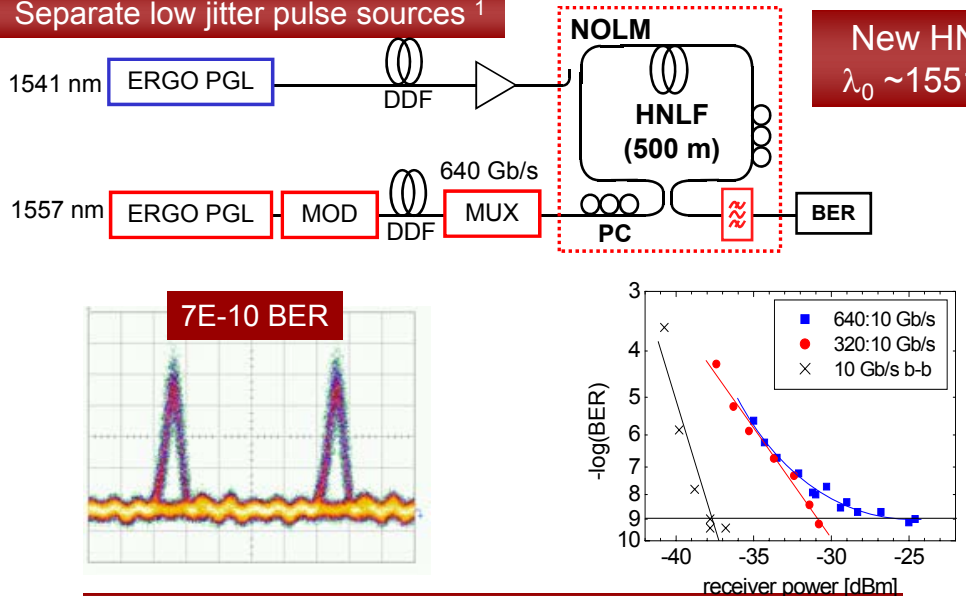

Error free 640/10 Gb/s: clear and open eyes

J. Seoane et al, ECOC 2004, paper We1.5.4.

L.K. Oxenloewe 
Nano-waveguides

Efficient FWM conversion
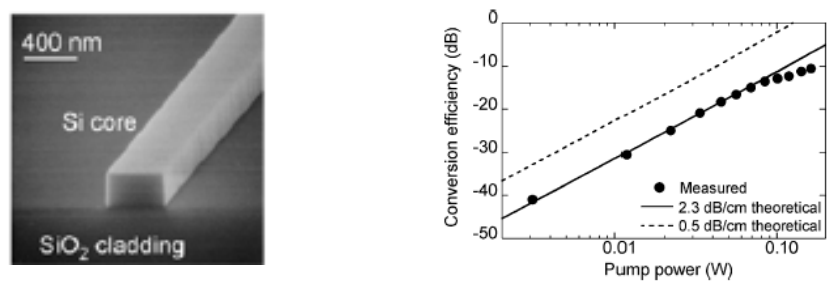

K. Yamada, IEEE Photon. Techn. Lett., Vol. 18, NO. 9, p. 1046, 2006

For extensive theoretical treatment of nonlinearities in Silicon waveguides see Q. Lin et al., Opt. Exp., 10 Dec. 2007, Vol. 15, No. 25.

\section{All-optical control on Si chip}

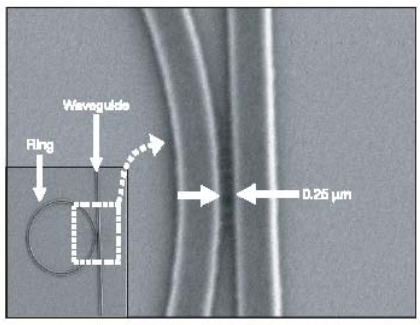

$\sim 500$ ps switching time $25 \mathrm{pJ}$ control pulses

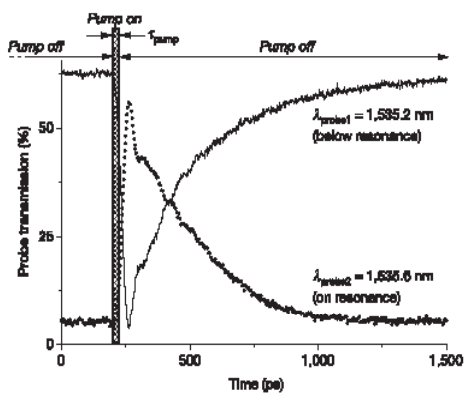

V.R. Almeida et al., NATURE, Vol. 431, p. 1081, Oct. 2004 


\section{All-optical switching in photonic crystal DTil}

\section{Concept based on Kerr nonlinearity}
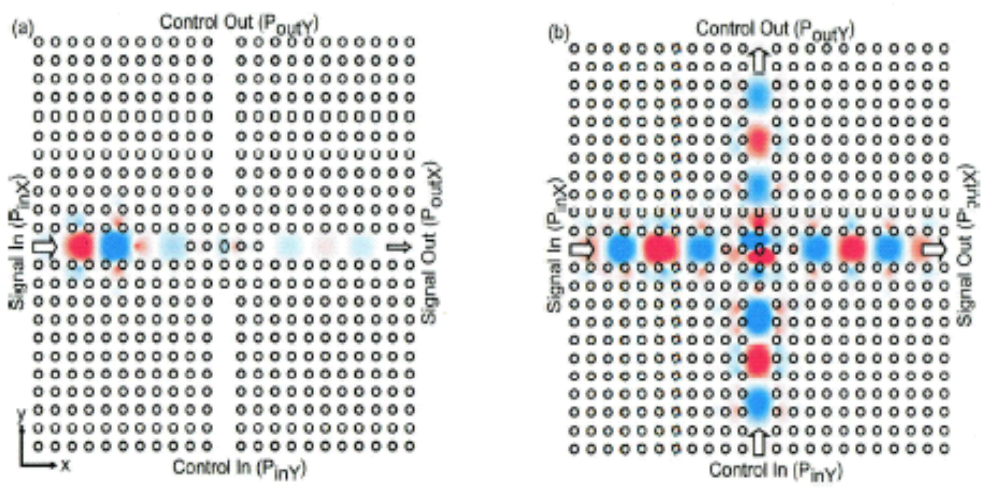

M.F. Yanik et al., OPTICS LETTERS,

Vol. 28, No. 24 / December 15, 2003

\section{Chalcogenide glass}

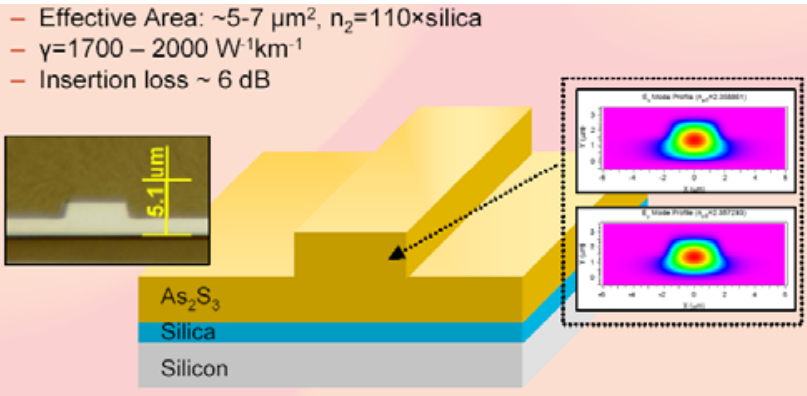

http://www.cudos.org.au/cudos/meetings/Astrophotonics/

160-Gb/s Optical Time-Division Demultiplexing by Four-Wave Mixing

M. D. Pelusi et al., PTL, vol. 19, NO. 19, Oct., 2007

$40 \mathrm{~Gb} / \mathrm{s}$ wavelength conversion by XPM

M.R.E. Lamont et al., Opt. Exp., Vol. 15, No. 23, Nov. 2007 
Nonlinear optical properties of third-order nonlinear materials @ $\lambda=1.5 \mu \mathrm{m}$.

\begin{tabular}{l|l|l|l} 
Material & $\begin{array}{l}n_{2} \\
{\left[\times 10^{-20} \mathrm{~m}^{2} / \mathrm{W}\right]}\end{array}$ & $\begin{array}{l}\alpha_{2} \\
{\left[10^{-12} \mathrm{~m} / \mathrm{W}\right]}\end{array}$ & FOM \\
\hline Chalcogenide glass $\left(\mathrm{As}_{2} \mathrm{~S}_{3}\right)$ & 290 & $<0.01$ & $>10$ \\
\hline $\begin{array}{l}\text { Chalcogenide glass } \\
\left(\mathrm{As}_{2} \mathrm{Se}_{3}\right)\end{array}$ & 1200 & 1 & 2 \\
\hline Bismuth Oxide $\left(\mathrm{Bi}_{2} \mathrm{O}_{3}\right)^{*}$ & 110 & - & - \\
\hline Silicon $(\mathrm{Si})$ & 440 & 8.4 & 0.4 \\
\hline Silica $\left(\mathrm{SiO}_{2}\right)^{*}$ & 2.2 & - & -
\end{tabular}

Vahid G. Ta'eed et al., Opt. Exp., Vol. 15, No. 15, 23 July 2007

Silicon-on-insulator waveguides $\mathrm{w}$. nonlinear cover

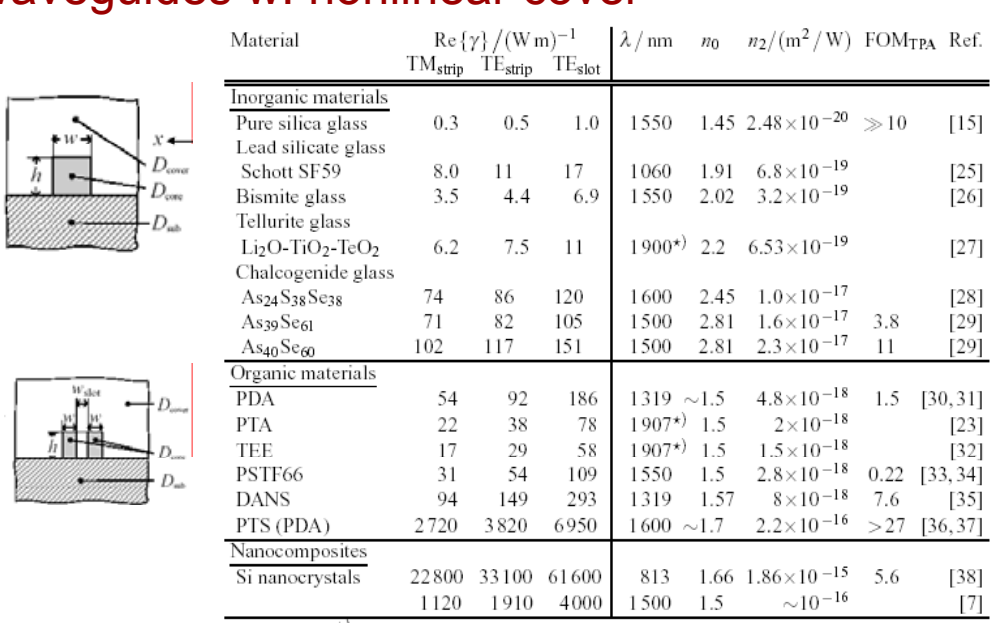

C. Koos et al., Opt. Exp., Vol. 15, No. 10, 14 May 2007. 
Fast waveguide with polymer cover
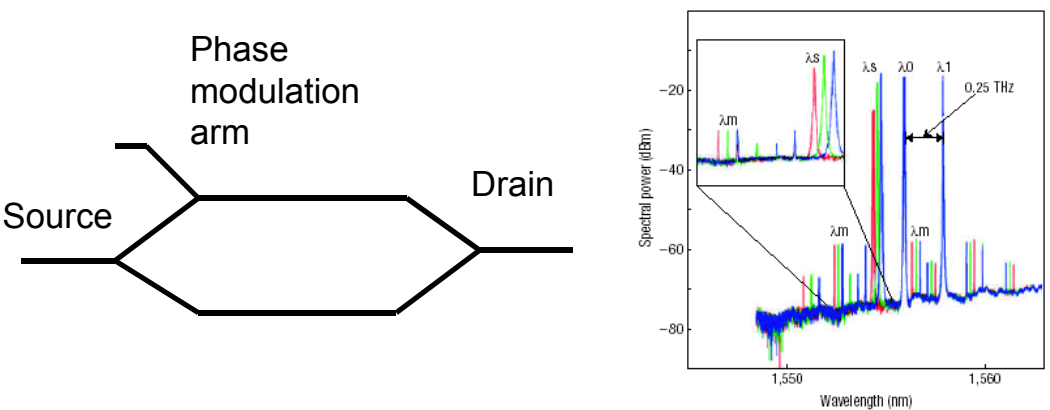

$\mathrm{THz}$ modulation speeds predicted

Michael Hochberg et al., Nature Materials. Vol. 5, p. 705, Sept. 2006

Second harmonic generation in PPLN with QPM

Signal light

Pump light

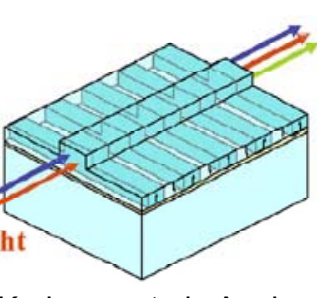

Sunao Kurimura et al., Appl. Phys. Lett. 89, 191123 (2006)

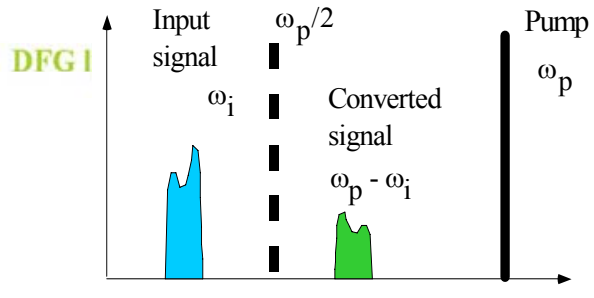

Frequency 
Experimental set-up for $\chi^{(2)}$ sumfrequency generation for $\mathrm{CR}$

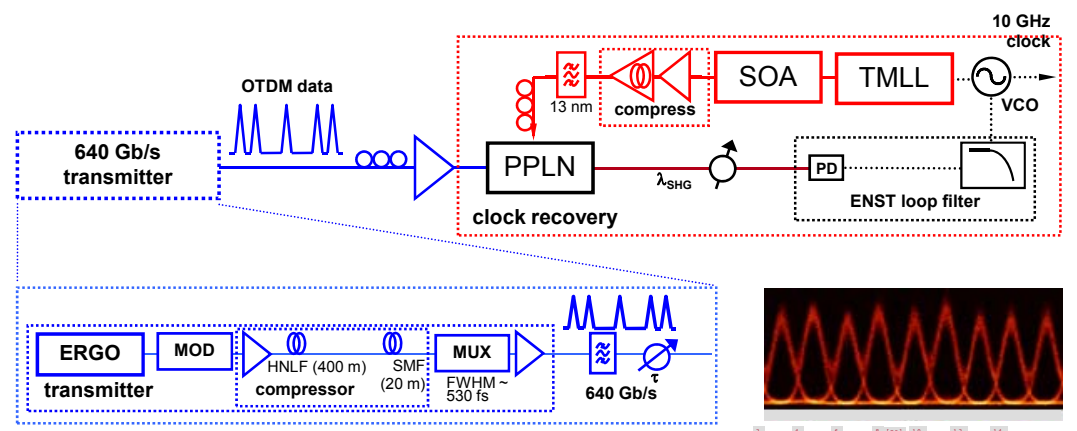

Soliton compression in fibre w. gain $\rightarrow$ tuneable FWHM: 400-1100 fs

Loop length $\sim 43 \mathrm{~m}+$ EDFA, but loop filter b.w. $\sim 200 \mathrm{kHz} \rightarrow$ ok

L.K. Oxenloewe et al, submitted for Electronics Letters

L.K. Oxenloewe

Switching/Demux highlights from litterature - I

- 640 to $10 \mathrm{Gbit} / \mathrm{s}$ using XPM in NOLM ${ }^{1}$

(1.28 Tb/s IM+pol-MUX)

- 640 to $10 \mathrm{Gbit} / \mathrm{s}$ using Kerr switching with parametric amplification ${ }^{2}$

- $640 \mathrm{Gbit} / \mathrm{s}$ in NOLM ${ }^{3}$ COM

- $640 \mathrm{~Gb} / \mathrm{s}^{4}(2.56 \mathrm{~Tb} / \mathrm{s}$ DQPSK + pol-MUX) - Weber

- 500 to $10 \mathrm{Gbit/s}$ using FWM ${ }^{5}$

\section{Fibres fastest - but - stability of lengthy fibres?}

1: M. Nakazawa, et al, Electron. Lett. 34 (9) 1998. 2: S. Watanabe et al, ECOC 2004, paper

Th4.1.6. 3: COM 4: Weber et al, Electron. Lett. 42 (3) 2006. 5: T. Morioka et al, Electron. Lett. 32

(9) (1996). TuH6. 


\section{OMV1.pdf}

\section{Switching/Demux highlights from litterature - II}

\section{Semiconductor switches}

- 336 to $10.5 \mathrm{Gbit} / \mathrm{s}$ using SOA based Mach-Zehnder interferometer ${ }^{6}$

- 160 to $10 \mathrm{Gbit/s}$ applying voltage control signal to EAM ${ }^{7}$

- 160 to $10 \mathrm{Gbit/s}$ using XAM in an EAM ${ }^{8}$

- 320 to $10 \mathrm{Gbit} / \mathrm{s}$ integrating ultra-fast photodiode and EAM ${ }^{9}$

- 640 to $10 \mathrm{Gbit} / \mathrm{s}$ using fa-XPM in an SOA ${ }^{10}$

Alternative switches

- $160 \mathrm{Gbit} / \mathrm{s}$ demux in chalcogenide waveguide $\left(\chi^{(3)}\right)^{\mathrm{X}}$, cuDOS

- $640 \mathrm{Gbit} / \mathrm{s}$ clock recovery with PPLN QPM-device $\left(\chi^{(2)}\right)$ XX, COM, ENST, NIMS,

- 16 Semiconductors slow - but - integration offers stability

$$
\text { Alternative switches fast - but - loss, dispersion }
$$

6: S. Nakamura et al, OFC 2002, paper FD3-1. 7: H. Chou et al, OFC 2003, paper ThX2. 8: L. Xu et al, ECOC 2004, paper We1.5.3, 9: S. Kodama et al, OFC 2003, paper ThX5. 10: E. Tangdiongga et al, PTL 18 (8) 2006640 G DEMUX W FA-XPM, X: M.D. Pelusi, ECOC-2007/PTL, XX: L.K. Oxenløwe et al, submitted to Electronics Letters

\section{L.K. Oxenloewe}

\begin{tabular}{|c|c|c|c|}
\hline Type & $\begin{array}{l}\text { Switching } \\
\text { energy }\end{array}$ & Timescale & Principle of operation/comments \\
\hline Transistor & $\begin{array}{l}\text { Switch: } 1 \mathrm{fJ} \\
\text { Circuit: } 10 \mathrm{pJ}\end{array}$ & Sub-ns & $\begin{array}{l}\text { CMOS electronic/digital. Extreme- } \\
\text { ly small footprint. Transport more } \\
\text { power-hungry than actual } \\
\text { switching action. }\end{array}$ \\
\hline $\begin{array}{l}\text { Nonlinear } \\
\text { directional } \\
\text { coupler }\end{array}$ & $<1 \mathrm{~nJ}$ & fs & $\begin{array}{l}\text { All-optical, analog switch. Ultra } \\
\text { fast (Kerr effect). } \\
\text { "Classical" nonlinear optics. }\end{array}$ \\
\hline $\begin{array}{l}\text { SOA-gate + } \\
\text { filter }\end{array}$ & $\sim 1-10 \mathrm{fJ}$ & ps & $\begin{array}{l}\text { Requires bias. Precise } \\
\text { wavelength adjustment }\end{array}$ \\
\hline SOA-MZI & $100 \mathrm{fJ}$ & ps & Requires bias. \\
\hline $\begin{array}{l}\text { Intersubband } \\
\text { abs. switch }\end{array}$ & $5-10 \mathrm{pJ}$ & ps & \\
\hline
\end{tabular}

Modified after TF Krauss, http://www.cfn.uni-karlsruhe.de/web/ 


\section{OMV1.pdf}

\begin{tabular}{|c|c|c|c|}
\hline Type & $\begin{array}{l}\text { Switching } \\
\text { energy }\end{array}$ & Timescale & Principle of operation/comments \\
\hline Transistor & $\begin{array}{l}\text { Switch: } 1 \mathrm{fJ} \\
\text { Circuit: } 10 \text { pJ }\end{array}$ & Sub-ns & $\begin{array}{l}\text { CMOS electronic/digital. Extreme- } \\
\text { ly small footprint. Transport more } \\
\text { power-hungry than actual } \\
\text { switching action. }\end{array}$ \\
\hline $\begin{array}{l}\text { Nonlinear } \\
\text { fiber }\end{array}$ & $1-10 \mathrm{pJ}$ & fs & $\begin{array}{l}\text { Long length require stable } \\
\text { packaging. }\end{array}$ \\
\hline $\begin{array}{l}\text { PC laser } \\
\text { cavity }\end{array}$ & $50-100 \mathrm{fJ}$ & ps & Precisely adjusted wavelength \\
\hline $\begin{array}{l}\text { Dual ring } \\
\text { flip-flop }\end{array}$ & $5 \mathrm{fJ}$ & ps & $\begin{array}{l}\text { Digital. Injection locking of } \\
\text { CW/ACW modes. Large bias (10's } \\
\text { of } m A \text { ) required }\end{array}$ \\
\hline Micro ring & $10 \mathrm{fJ}$ & ns & Analog. Carrier injection. \\
\hline
\end{tabular}

Modified after TF Krauss, http://www.cfn.uni-karlsruhe.de/web/

\section{Switching Power-Delay Characteristics}

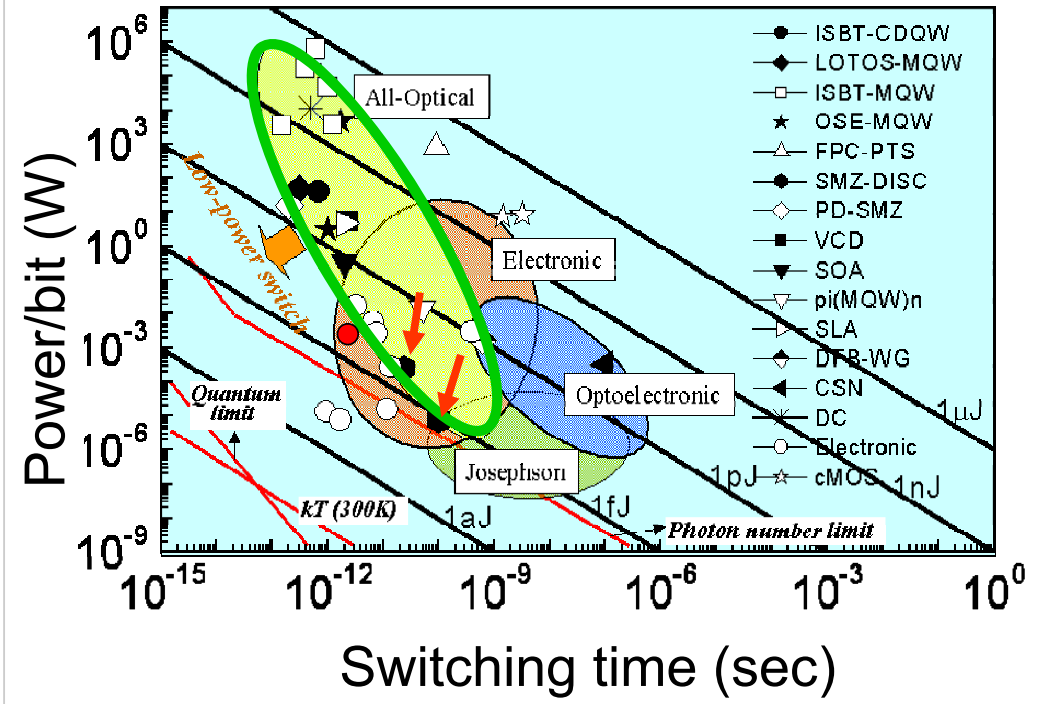

O. Wada, FESTA 


\section{The principle of XOR in an IWC}
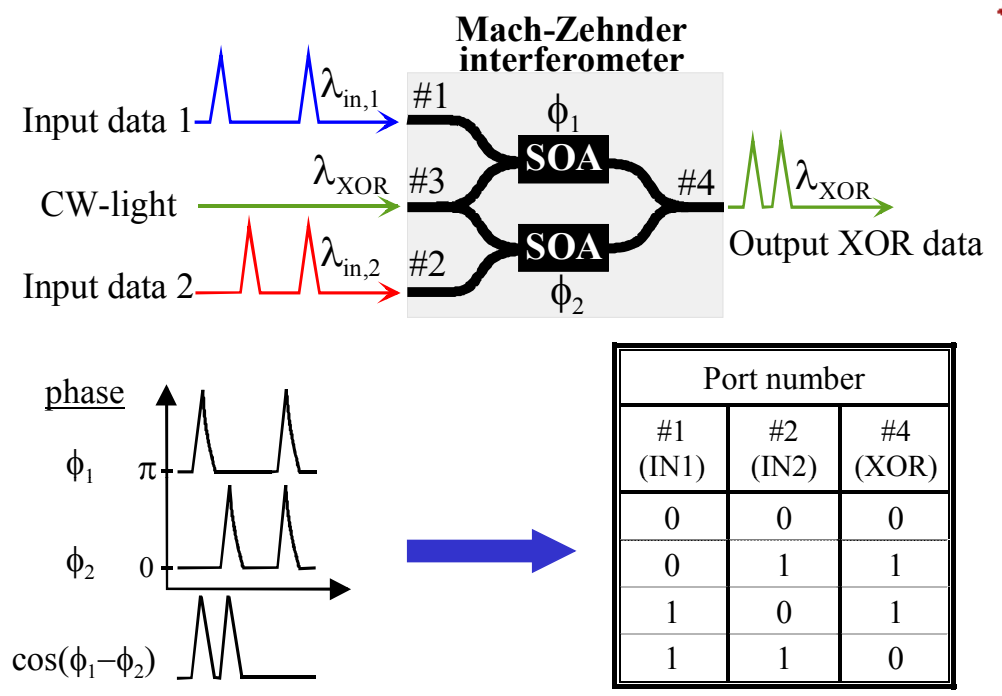

Tina Fjelde

40 Gbit/s all-optical XOR gate

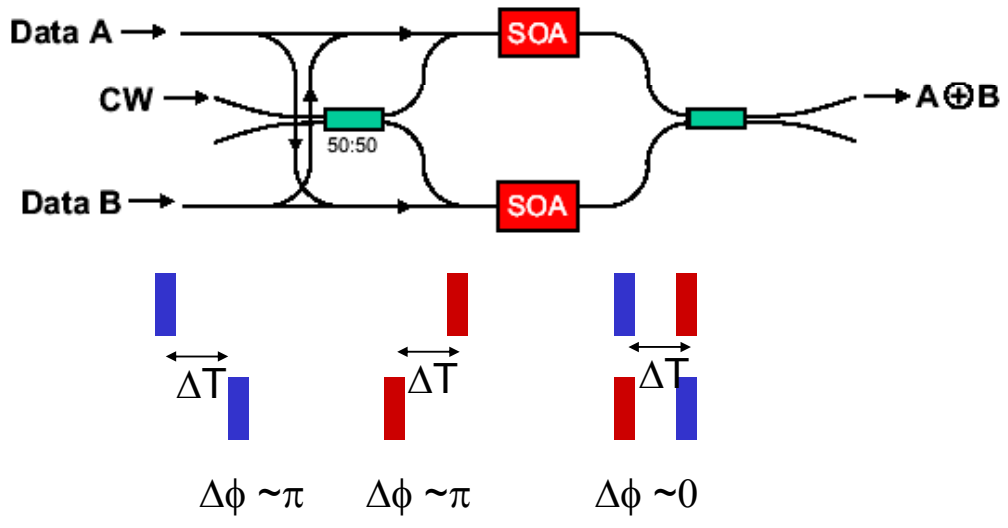

R.P. Webb et al., Electron. Lett. VoL 39 No. 1, 2003 
Header swapping using IWC

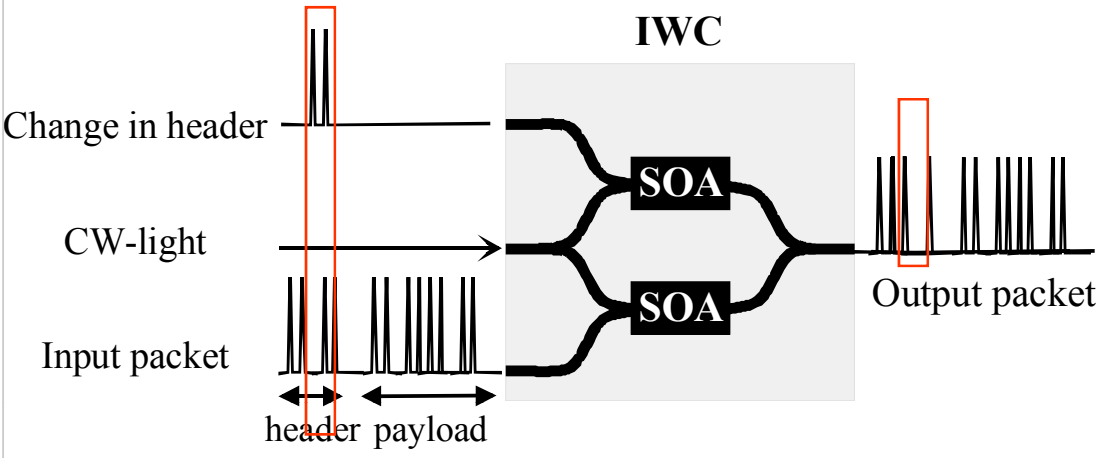

Tina Fjelde

Binary counter using all-optical logic gates

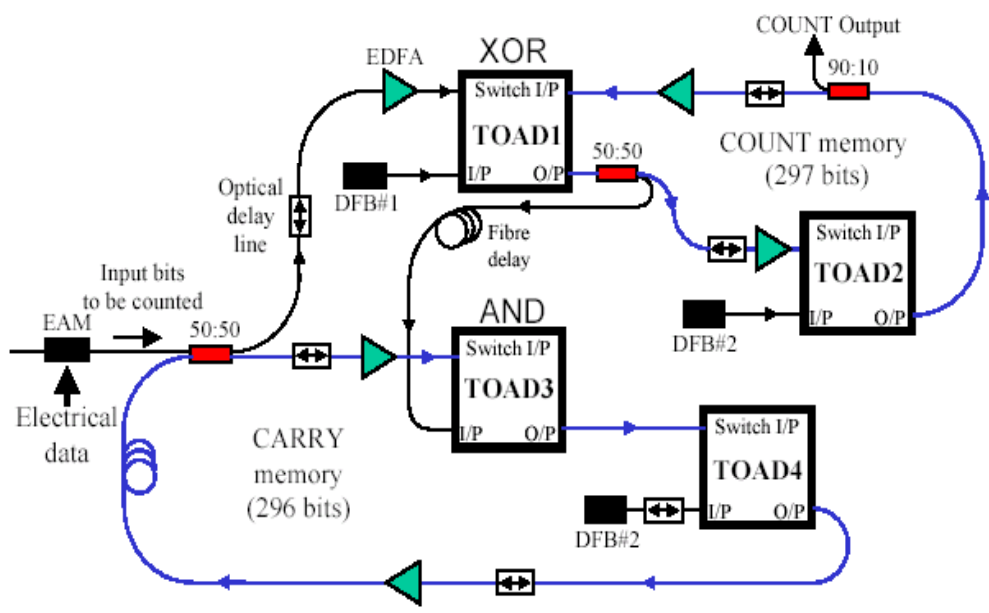

A. Poustie et al., Opt. Exp., Vol. 6, No. 3, 31 January 2000 


\section{All-Optical 40-Gb/s Packet Routing using two UNI-gates}

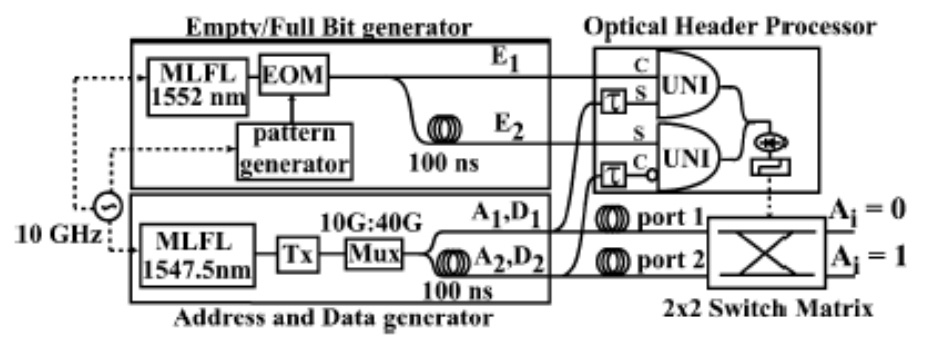

J.P. Wang et al., PTL, Vol. 18, NO. 21, p. 2275, Nov., 2006

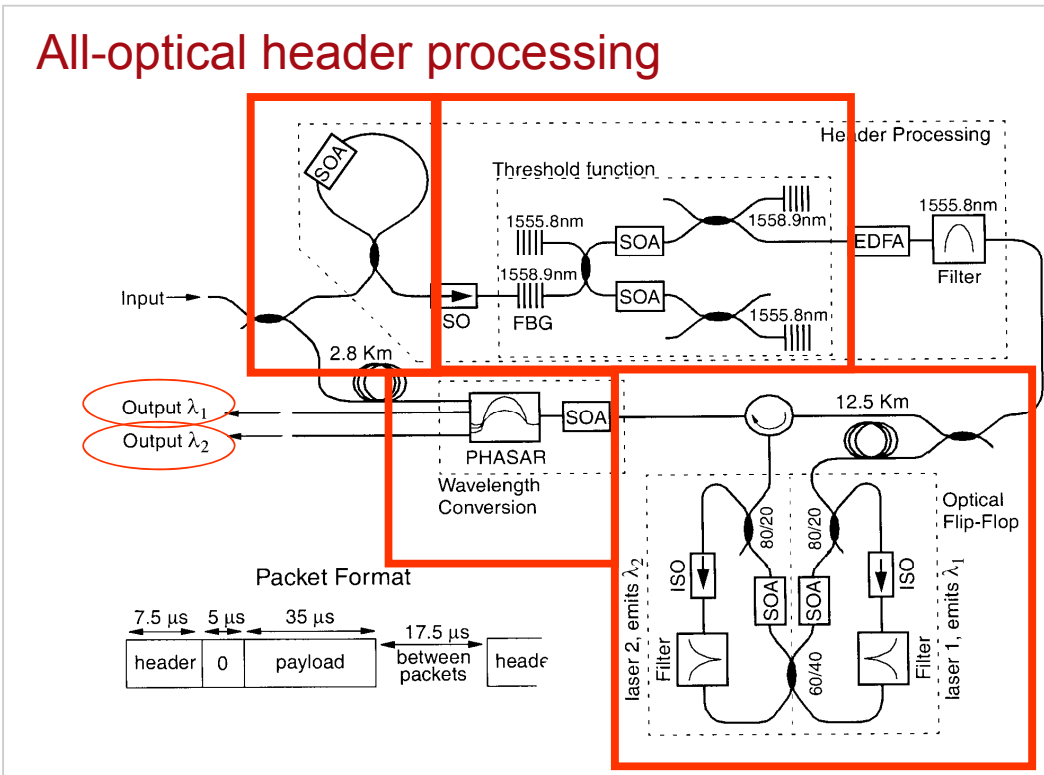

M.T. Hill et al., Electron. Lett., p. 774, 2001 


\section{All-Optical Flip-Flop \\ based on Coupled Ring Lasers}

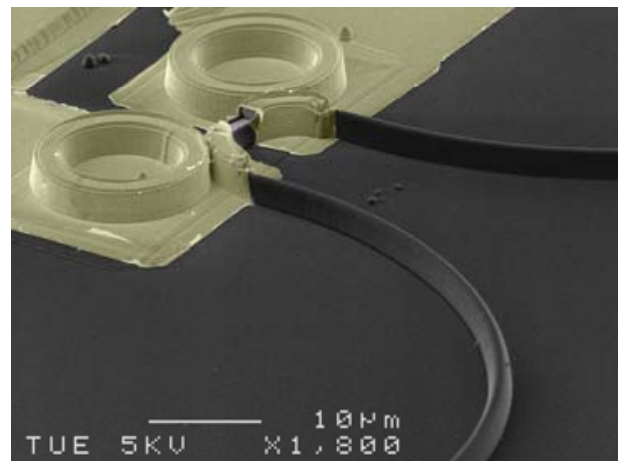

- Continuous lasing for $16 \& 12 \mu \mathrm{m}$ diameter

- Threshold current $\sim 5 \mathrm{~mA}$

M.T. Hill et al, Nature 432, 206-209 (2004)

\section{Experimental Results}
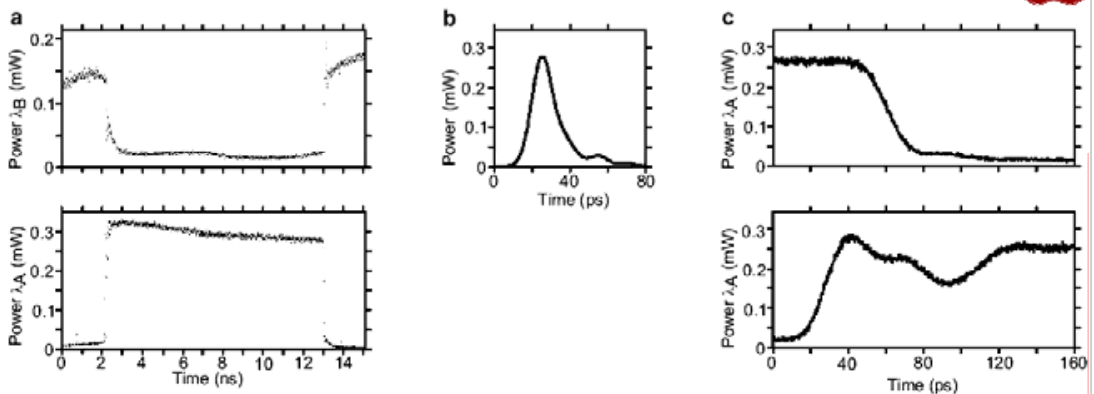

- Pulsed current operation for devices with $\sim 1 \%$ coupling to waveguide (coupling introduced $50 \%$ loss)

- Achieved bistability, $10-90 \%$ transition time $<18$ ps, $5.5 \mathrm{fJ}$ switch pulse energy

- Switching with $2 \mathrm{fJ}$ also possible

M.T. Hill et al, Nature 432, 206-209 (2004) 
All-optical flip-flops based on Mach-Zhender structures

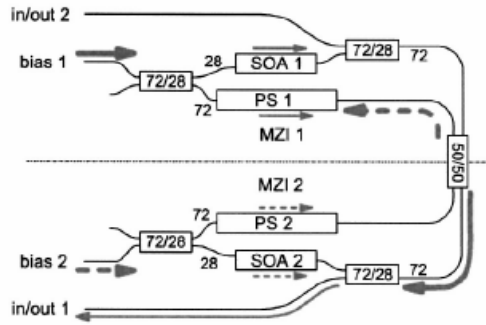

M.T. Hill et al., Opt. Lett., Vol. 30, No. 13, July 1, 2005

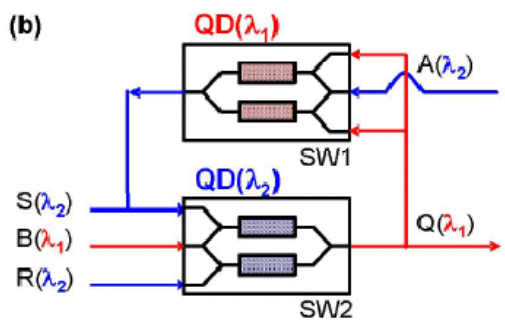

N. Ozaki, 19th IPRM, May 2007 Matsue, Japan

Flip-flop with SOA and DFB laser

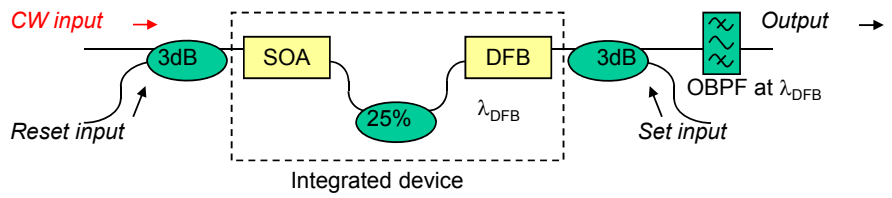

- Repetition rate: $1.25 \mathrm{GHz}$ (set-to-set)

- Set/reset pulse width: 50 ps

- Set/reset energy: < $1.6 \mathrm{pJ}$

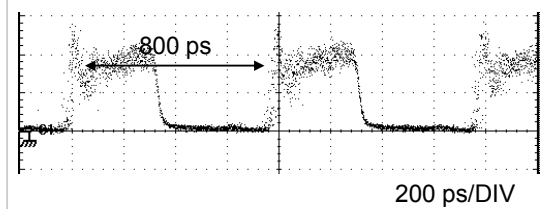

- Dynamic contrast ratio: $19 \mathrm{~dB}$

- Switching time: $\sim 50$ ps

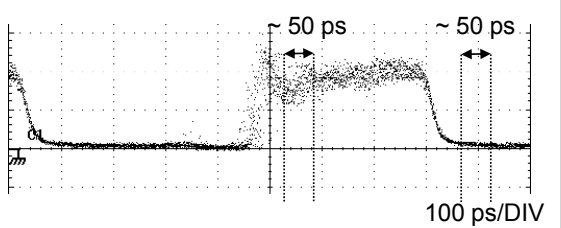

W. D'Oosterlinck et al., Opt. Exp., Vol. 15, No. 10, 14 May 2007

J. Buron and F. Öhman 


\section{Micro and Nanolasers for digital} photonics

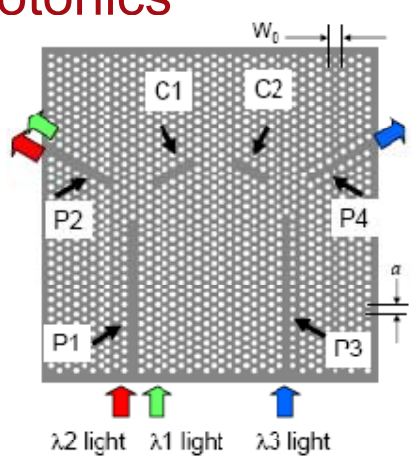

Coupled two-port resonant tunneling filter

A. Shinya et al., Opt. Exp., Vol. 14, No. 3, February 2006
Levels of reflection and isolation between inputoutput a challenge

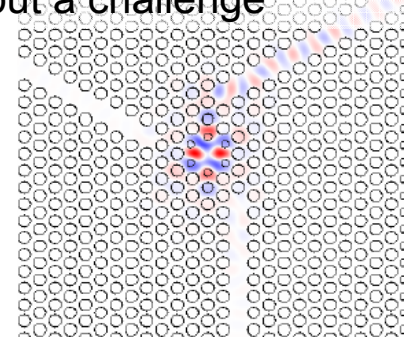

Martin T. Hill, in Proc. of ECIO, paper WCO, Copenhagen Denmark April 2007

\section{OITDA Technology roadmaps}

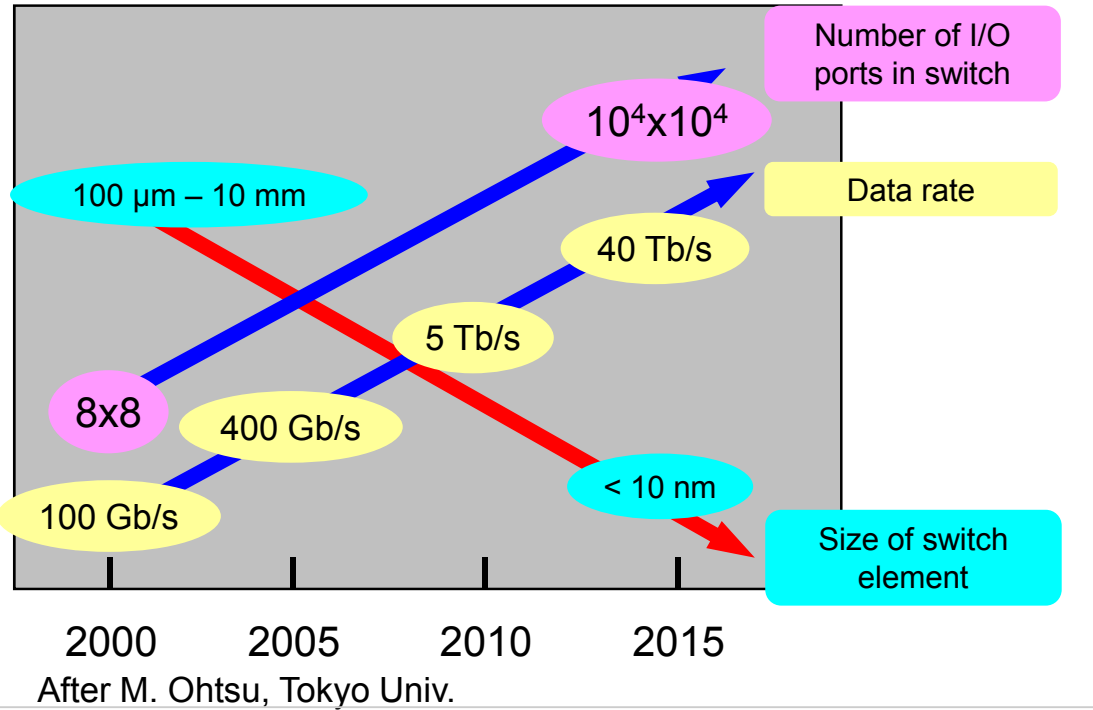




\section{OMV1.pdf}
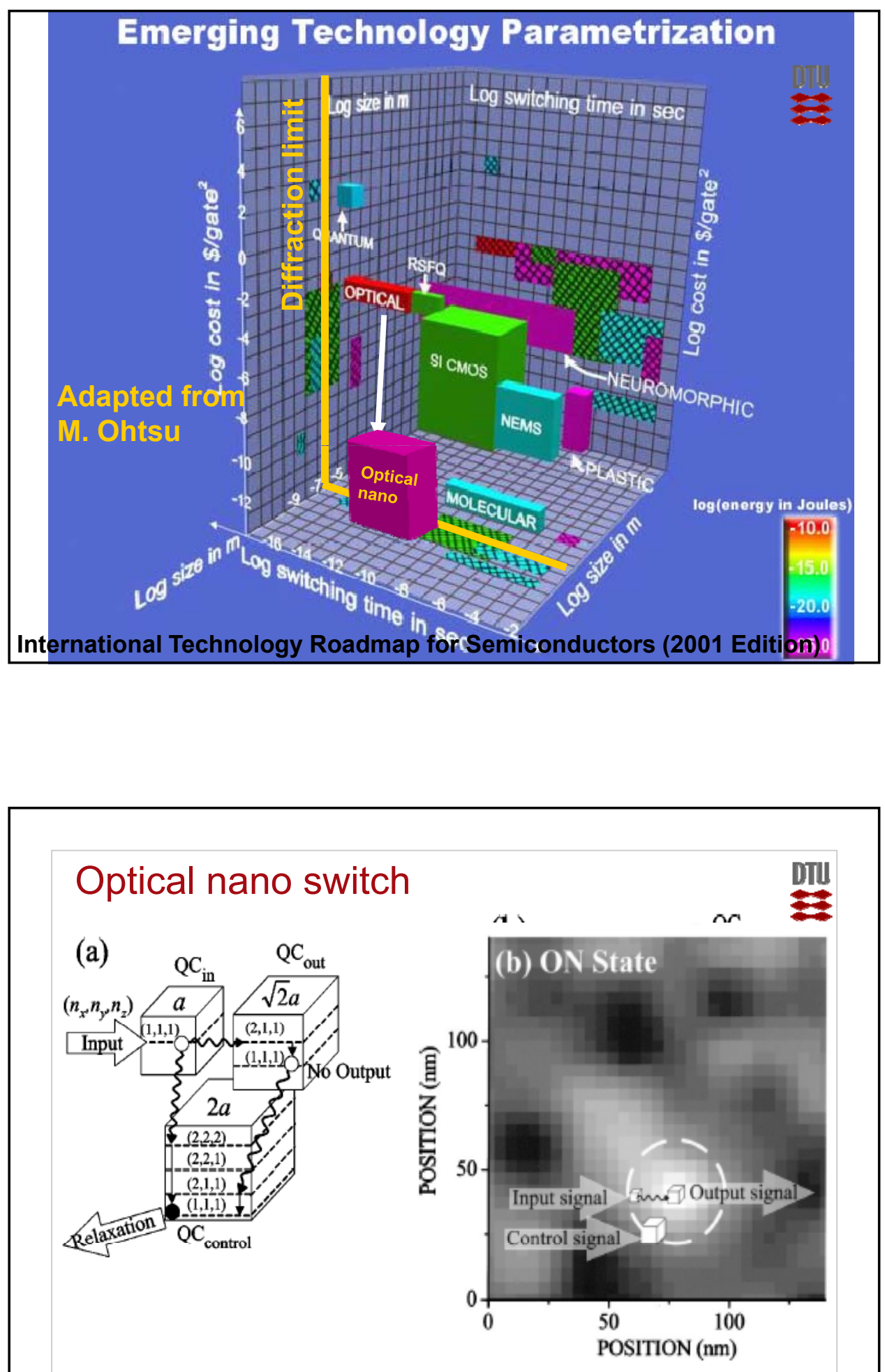

Kawazoe et al., Appl. Phys. Lett., Vol. 82, No. 18, 5 May 2003 
Lithographically controlled, seeded nanowire growth of photonic crystals \& other 2D arrays
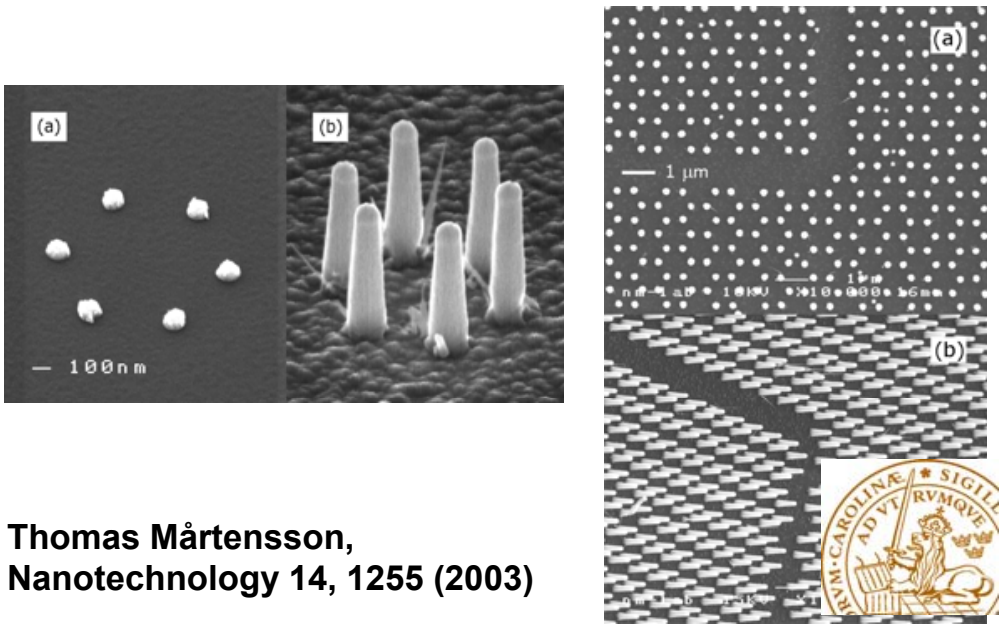

Thomas Mårtensson, Nanotechnology 14, 1255 (2003)

\section{Plasmonic interaction}
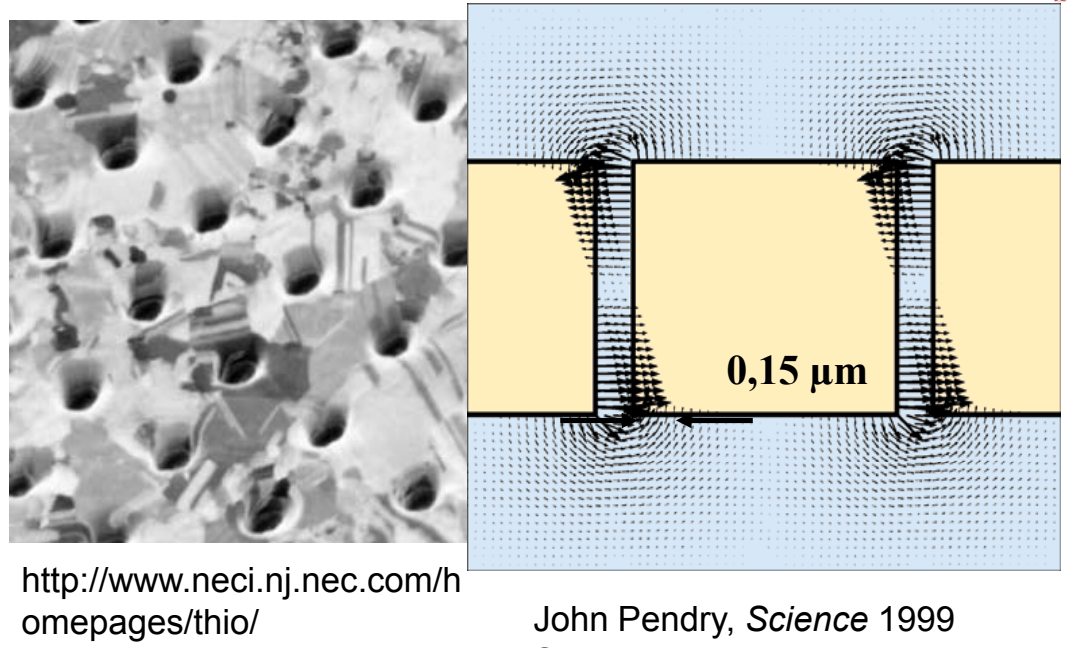

John Pendry, Science 1999

September 10; 285: 1687-1688 


\section{Integrated optics using surface plasmon polaritons}

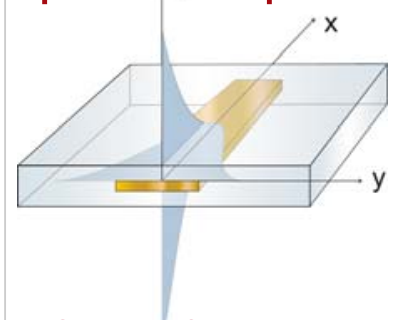

\section{Metal thickness:}

$\sim 15 \mathrm{~nm}$

R. Charbonneau, et. al., Optics Express 13, 977 (2005)

A. Boltasseva, et. al., J. Lightwave Technol. 23, 413 (2005)

Control of propagation and dispersion by structuring of metal
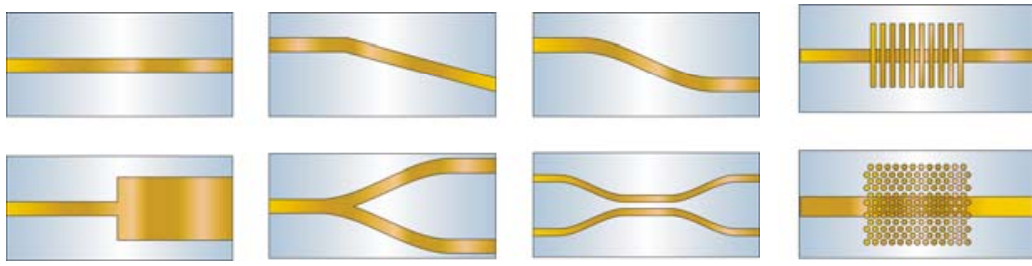

Alexandra Boltasseva, COM

\section{Single Photon Transistor}

Single photon generation and nonlinear optics with surface plasmons Chang, Sørensen et al.,Nature Physics Vol. 3, p. 807, Nov. 2007

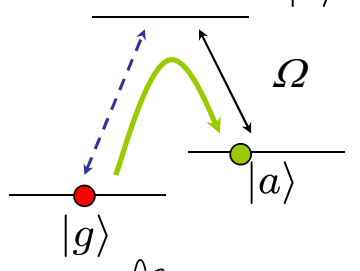

$|e\rangle$ 


\section{Conclusions}

Nonlinear elements based on SOA, fibers and waveguide structures have capabilities of simple processing at data rates of $100-600 \mathrm{~Gb} / \mathrm{s}$

- Switching powers comparable to electronics

- No clear preference for technology at this point of time

Higher packing density needed in future systems Fast progress in highly nonlinear engineered materials Photonic Bandgap Structures and nanowaveguides have potential for more compact photonic circuits - but more work needed

\section{Conclusions cont.}

Nanostructures in switching at the very beginning Plasmons may be useful for more compact photonic signal processing elements More experiments needed

Exciting new technologies

Closer to fundamental limits Engineering of material properties 